\title{
THE JOINT LAPLACE TRANSFORMS FOR DIFFUSION OCCUPATION TIMES
}

\author{
BIN LI, ${ }^{*}$ University of Iowa \\ XIAOWEN ZHOU,** Concordia University
}

\begin{abstract}
In this paper we adopt the perturbation approach of Landriault, Renaud and Zhou (2011) to find expressions for the joint Laplace transforms of occupation times for time-homogeneous diffusion processes. The expressions are in terms of solutions to the associated differential equations. These Laplace transforms are applied to study ruin-related problems for several classes of diffusion risk processes.
\end{abstract}

Keywords: Exit problem; occupation time; ruin probability; time-homogeneous diffusion

2010 Mathematics Subject Classification: Primary 60J60

Secondary 60G17

\section{Introduction}

The occupation time is the amount of time a stochastic process stays within a certain range. It is an interesting topic for stochastic processes. Many explicit results on Laplace transforms for occupation times have been obtained for some well-known examples of diffusion processes; see, e.g. [6] for a collection of such results. Some results related to occupation times for general diffusion processes can be found in [18] and [19]. More recently, Laplace transforms have also been obtained for occupation times of processes with jumps; see [7], [9], [14], [15], [17], [23], and [24] for results along this line and their applications in risk theory and finance. To the best of the authors' knowledge, joint Laplace transforms have not been studied systematically for general diffusion processes.

The standard way of finding the Laplace transform for occupation times is to solve the associated (integro-) differential equation via the Feynman-Kac representation. The excursion theory has also been applied to obtain occupation time related Laplace transforms; see [18] and [19]. An alternative perturbation approach was recently proposed in [15] for the spectrally negative Lévy processes. With this approach, the Laplace transforms for occupation times can be obtained by an approximation argument based on the solutions to the exit problems, which is an excursion theory argument at its heart. Since the exit problems for time-homogeneous diffusion processes can also be solved explicitly, in this paper we adopt the strategy of [15] to study the joint Laplace transforms for diffusion occupation times. These Laplace transforms are expressed in terms of solutions to the differential equation associated to the diffusion generator.

The results in this paper find applications in the study of ruin problems for the so-called diffusion risk process with random observation times and the so-called omega model, where,

Received 13 July 2012; revision received 22 February 2013.

* Current address: Department of Statistics and Actuarial Science, University of Waterloo, 200 University Avenue West, Waterloo, Ontario, N2L 3G1, Canada. Email address: bin.li@uwaterloo.ca

** Postal address: Department of Mathematics and Statistics, Concordia University, 1455 de Maisonneuve Blvd. West, Montreal, Quebec, H3G 1M8, Canada. Email address: xiaowen.zhou@concordia.ca 
for both models, the ruin probability can be expressed in terms of its occupation time. Our results can also be applied to study the Brownian surplus process with two-valued drift.

The rest of the paper is arranged as follows. In Section 2 we introduce the time-homogeneous diffusion processes and their exit problems. In Section 3, the desired Laplace transforms of diffusion occupation times are found. In Section 4, possible applications of the results obtained in Section 3 to several diffusion risk processes are discussed. In Section 5, explicit expressions are presented for the examples of Brownian motion with drift and Brownian motion with twovalued drift.

\section{Time-homogeneous diffusion processes}

We now introduce the one-dimensional diffusion process $X$ considered in this paper. For $-\infty \leq l_{1}<l_{2} \leq \infty$, write $I$ for the interval with endpoints $l_{1}$ and $l_{2}$. For simplicity, we consider only the case in which both $l_{1}$ and $l_{2}$ are inaccessible, i.e. neither of them can be reached in finite time. In the rest of paper, we mostly choose $l_{1}=-\infty$ and $l_{2}=\infty$. The $I$-valued regular time-homogeneous diffusion process $X=\left\{X_{t}, t \geq 0\right\}$, defined on a filtered probability space $\left\{\Omega, \mathbb{P},\left\{\mathcal{F}_{t}, t \geq 0\right\}\right\}$, is specified by the stochastic differential equation

$$
\mathrm{d} X_{t}=\mu\left(X_{t}\right) \mathrm{d} t+\sigma\left(X_{t}\right) \mathrm{d} W_{t},
$$

where $X_{0}=x_{0}$ is the initial value and $\left\{W_{t}, t \geq 0\right\}$ is a standard Brownian motion. Throughout the paper, we assume that (2.1) allows a unique strong solution, which is guaranteed if there exists a constant $K>0$ such that, for all $x, y \in I$,

$$
|\mu(x)-\mu(y)|+|\sigma(x)-\sigma(y)| \leq K|x-y|, \quad \mu^{2}(x)+\sigma^{2}(x) \leq K^{2}\left(1+x^{2}\right) .
$$

Let $T_{x}=\inf \left\{t \geq 0: X_{t}=x\right\}$ be the first hitting time of $X$ at level $x$ with the convention that inf $\varnothing=\infty$. The Laplace transforms associated with the two-sided exit problem for the diffusion process $X$ were first solved in [8]. Suppose that $g_{-, r}(\cdot)$ and $g_{+, r}(\cdot)$ are two independent, positive, and convex solutions of the equation

$$
\frac{1}{2} \sigma^{2}(x) g^{\prime \prime}(x)+\mu(x) g^{\prime}(x)=r g(x), \quad r>0,
$$

with $g_{-, r}(\cdot)$ strictly decreasing and $g_{+, r}(\cdot)$ strictly increasing. For many particular diffusions of interest, the differential equation (2.3) yields explicit expressions for $g_{-, r}(\cdot)$ and $g_{+, r}(\cdot)$; see [6]. Define a pair of Laplace exponents

$$
\psi_{r}^{ \pm}(\cdot)= \pm \frac{g_{ \pm, r}^{\prime}(\cdot)}{g_{ \pm, r}(\cdot)}, \quad r>0 .
$$

In particular, denoting by

$$
G(x)=\exp \left[-\int^{x} \frac{2 \mu(y)}{\sigma^{2}(y)} \mathrm{d} y\right],
$$

and letting $r \rightarrow 0+$, we choose

$$
g_{-, 0}(x) \begin{cases}\equiv 1 & \text { if } \int_{x}^{\infty} G(y) \mathrm{d} y=\infty, \\ =\int_{x}^{\infty} G(y) \mathrm{d} y & \text { otherwise, }\end{cases}
$$


and

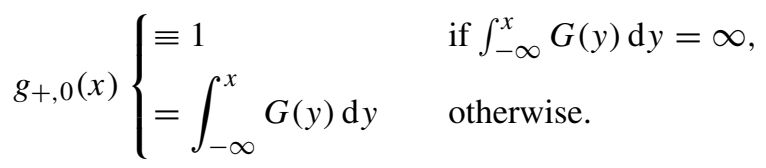

Hence,

$$
\psi_{0}^{-}(x)=\frac{G(x)}{\int_{x}^{\infty} G(y) \mathrm{d} y} \quad \text { and } \quad \psi_{0}^{+}(x)=\frac{G(x)}{\int_{-\infty}^{x} G(y) \mathrm{d} y} .
$$

Furthermore, for $r>0$, define a function

$$
f_{r}(y, z)=g_{-, r}(y) g_{+, r}(z)-g_{-, r}(z) g_{+, r}(y) .
$$

Note that the function $f_{r}(y, z)$ is strictly decreasing in $y$ and strictly increasing in $z$, and $f_{r}(y, z)=0$ if and only if $y=z$. For ease of notation, denote by

$$
f_{1, r}(y, z)=\frac{\partial}{\partial y} f_{r}(y, z), \quad f_{2, r}(y, z)=\frac{\partial}{\partial z} f_{r}(y, z), \quad \text { and } \quad f_{12, r}(y, z)=\frac{\partial^{2}}{\partial y \partial z} f_{r}(y, z) \text {. }
$$

Throughout the paper, for ease of notation, we write $\mathbb{E}_{x_{0}}[\cdot]=\mathbb{E}\left[\cdot \mid X_{0}=x_{0}\right]$ for the conditional expectation, $\mathbb{P}_{x_{0}}\{\cdot\}$ for the corresponding probability, and $\mathbb{E}_{x_{0}}[\cdot ; C]=\mathbb{E}_{x_{0}}\left[\cdot \mathbf{1}_{C}\right]$ with $\mathbf{1}_{C}$ denoting the indicator function of a set $C \subset \Omega$. Furthermore, we drop the subscript indicating the initial value if $x_{0}=0$.

For $a<x<b$, we have

$$
\mathbb{E}_{x}\left[\mathrm{e}^{-r T_{a}} ; T_{a}<T_{b}\right]=\frac{f_{r}(x, b)}{f_{r}(a, b)} \quad \text { and } \quad \mathbb{E}_{x}\left[\mathrm{e}^{-r T_{b}} ; T_{b}<T_{a}\right]=\frac{f_{r}(a, x)}{f_{r}(a, b)} .
$$

In particular, letting $r \rightarrow 0+$ in (2.9) yields the two-sided exit probabilities

$$
\mathbb{P}_{x}\left\{T_{b}<T_{a}\right\}=\frac{\int_{a}^{x} G(y) \mathrm{d} y}{\int_{a}^{b} G(y) \mathrm{d} y} \quad \text { and } \quad \mathbb{P}_{x}\left\{T_{a}<T_{b}\right\}=\frac{\int_{x}^{b} G(y) \mathrm{d} y}{\int_{a}^{b} G(y) \mathrm{d} y} ;
$$

see, e.g. [6], [10], and [13]. Moreover, for $r>0$, we have

$$
\lim _{x \rightarrow-\infty} g_{-, r}(x)=\lim _{x \rightarrow \infty} g_{+, r}(x)=\infty \quad \text { and } \quad \lim _{x \rightarrow \infty} g_{-, r}(x)=\lim _{x \rightarrow-\infty} g_{+, r}(x)=0 .
$$

In addition,

$$
\lim _{r \rightarrow \infty} \psi_{r}^{ \pm}(\cdot)=\infty
$$

see Equation (12) of [19]. Therefore, letting $b \rightarrow \infty$ in the first relation of (2.9) and $a \rightarrow-\infty$ in the second relation of (2.9), for $a<x<b$, we have

$$
\mathbb{E}_{x} \mathrm{e}^{-r T_{a}}=\frac{g_{-, r}(x)}{g_{-, r}(a)} \quad \text { and } \quad \mathbb{E}_{x} \mathrm{e}^{-r T_{b}}=\frac{g_{+, r}(x)}{g_{+, r}(b)} .
$$

In the following, we collect some identities that will be used later. They can be easily verified by (2.4) and (2.8).

Lemma 2.1. For any $x \neq y$ and $r>0$, we have

$$
\begin{gathered}
f_{2, r}(x, x) f_{1, r}(y, y)-f_{2, r}(x, y) f_{1, r}(x, y)=-f_{12, r}(x, y) f_{r}(x, y), \\
-f_{1, r}(x, y) \psi_{r}^{-}(y)-f_{2, r}(x, y) \psi_{r}^{-}(x)=f_{12, r}(x, y)+f_{r}(x, y) \psi_{r}^{-}(x) \psi_{r}^{-}(y),
\end{gathered}
$$

and

$$
f_{1, r}(x, y) \psi_{r}^{+}(y)+f_{2, r}(x, y) \psi_{r}^{+}(x)=f_{12, r}(x, y)+f_{r}(x, y) \psi_{r}^{+}(x) \psi_{r}^{+}(y) .
$$




\section{The occupation times for diffusion processes}

Throughout the paper, denote by $e_{\delta}$ an independent, exponential random variable with rate $\delta>0$ and assume that $\lambda, \lambda_{1}, \lambda_{2}>0$. We first solve for $\mathbb{E} \exp \left[-\lambda \int_{0}^{e_{\delta}} \mathbf{1}_{\left\{X_{s}<0\right\}} \mathrm{d} s\right]$ using the perturbation approach from [15].

Theorem 3.1. It holds that

$$
\mathbb{E} \exp \left[-\lambda \int_{0}^{e_{\delta}} \mathbf{1}_{\left\{X_{s}<0\right\}} \mathrm{d} s\right]=\frac{\delta \psi_{\delta+\lambda}^{+}(0) /(\delta+\lambda)+\psi_{\delta}^{-}(0)}{\psi_{\delta+\lambda}^{+}(0)+\psi_{\delta}^{-}(0)} .
$$

Proof. Define an approximation $L_{\varepsilon}^{\delta}$ for the occupation time $\int_{0}^{e_{\delta}} \mathbf{1}_{\left\{X_{s}<0\right\}} \mathrm{d} s$ intuitively as follows. Up to time $e_{\delta}, L_{\varepsilon}^{\delta}$ counts both the time $X$ spends below level 0 and the time it takes to move from level 0 to level $\varepsilon$, but not from $\varepsilon$ to 0 . To make it rigorous, let $\theta$ be the shift operator such that $X_{t} \circ \theta_{s}=X_{s+t}$. Since $X$ starts from 0 , we put $T_{0}^{1}=0, T_{\varepsilon}^{1}=T_{\varepsilon}$, and

$$
T_{0}^{n+1}=T_{\varepsilon}^{n}+T_{0} \circ \theta_{T_{\varepsilon}^{n}}, \quad T_{\varepsilon}^{n+1}=T_{0}^{n+1}+T_{\varepsilon} \circ \theta_{T_{0}^{n+1}}, \quad \text { for } n \geq 1 .
$$

Then

$$
L_{\varepsilon}^{\delta}=\sum_{n=1}^{\infty}\left(T_{\varepsilon}^{n} \wedge e_{\delta}-T_{0}^{n} \wedge e_{\delta}\right)
$$

Hence, by the memoryless property we obtain

$$
\begin{aligned}
\mathbb{E}^{-\lambda L_{\varepsilon}^{\delta}} & =\mathbb{E}\left[\mathrm{e}^{-\lambda L_{\varepsilon}^{\delta}} ; T_{\varepsilon}<e_{\delta}\right]+\mathbb{E}\left[\mathrm{e}^{-\lambda \mathrm{e}_{\delta}} ; e_{\delta}<T_{\varepsilon}\right] \\
& =\mathbb{E}\left[\mathrm{e}^{-\lambda T_{\varepsilon}} ; T_{\varepsilon}<e_{\delta}\right] \mathbb{E}_{\varepsilon} \mathrm{e}^{-\lambda L_{\varepsilon}^{\delta}}+\mathbb{E} \mathrm{e}^{-\lambda \mathrm{e}_{\delta}}-\mathbb{E}\left[\mathrm{e}^{-\lambda \mathrm{e}_{\delta}} ; T_{\varepsilon}<e_{\delta}\right] \\
& =\mathbb{E} \mathrm{e}^{-(\delta+\lambda) T_{\varepsilon}} \mathbb{E}_{\varepsilon} \mathrm{e}^{-\lambda L_{\varepsilon}^{\delta}}+\frac{\delta}{\delta+\lambda}\left(1-\mathbb{E}^{-(\delta+\lambda) T_{\varepsilon}}\right) \\
& =\frac{g_{+, \delta+\lambda}(0)}{g_{+, \delta+\lambda}(\varepsilon)} \mathbb{E}_{\varepsilon} \mathrm{e}^{-\lambda L_{\varepsilon}^{\delta}}+\frac{\delta}{\delta+\lambda}\left(1-\frac{g_{+, \delta+\lambda}(0)}{g_{+, \delta+\lambda}(\varepsilon)}\right),
\end{aligned}
$$

where the last step is due to (2.12). Similarly, we have

$$
\begin{aligned}
\mathbb{E}_{\varepsilon} \mathrm{e}^{-\lambda L_{\varepsilon}^{\delta}} & =\mathbb{E}_{\varepsilon}\left[\mathrm{e}^{-\lambda L_{\varepsilon}^{\delta}} ; T_{0}<e_{\delta}\right]+\mathbb{E}_{\varepsilon}\left[\mathrm{e}^{-\lambda L_{\varepsilon}^{\delta}} ; e_{\delta}<T_{0}\right] \\
& =\mathbb{P}_{\varepsilon}\left\{T_{0}<e_{\delta}\right\} \mathbb{E} \mathrm{e}^{-\lambda L_{\varepsilon}^{\delta}}+\mathbb{P}_{\varepsilon}\left\{e_{\delta}<T_{0}\right\} \\
& =\mathbb{E}_{\varepsilon} \mathrm{e}^{-\delta T_{0}} \mathbb{E}^{-\lambda L_{\varepsilon}^{\delta}}+1-\mathbb{E}_{\varepsilon} \mathrm{e}^{-\delta T_{0}} \\
& =\frac{g_{-, \delta}(\varepsilon)}{g_{-, \delta}(0)} \mathbb{E}^{-\lambda L_{\varepsilon}^{\delta}}+1-\frac{g_{-, \delta}(\varepsilon)}{g_{-, \delta}(0)}
\end{aligned}
$$

Substituting (3.2) into (3.1), solving for $\mathbb{E}^{-\lambda L_{\varepsilon}^{\delta}}$, and taking the limit, we obtain

$$
\begin{aligned}
\mathbb{E} \exp \left[-\lambda \int_{0}^{e_{\delta}} \mathbf{1}_{\left\{X_{s}<0\right\}} \mathrm{d} s\right]= & \lim _{\varepsilon \rightarrow 0+} \mathbb{E}^{-\lambda L_{\varepsilon}^{\delta}} \\
= & \lim _{\varepsilon \rightarrow 0+}\left\{\left[\frac{\delta}{\delta+\lambda}\left(1-\frac{g_{+, \delta+\lambda}(0)}{g_{+, \delta+\lambda}(\varepsilon)}\right)+\frac{g_{+, \delta+\lambda}(0)}{g_{+, \delta+\lambda}(\varepsilon)}\left(1-\frac{g_{-, \delta}(\varepsilon)}{g_{-, \delta}(0)}\right)\right]\right. \\
& \left.\quad \times\left[1-\frac{g_{+, \delta+\lambda}(0)}{g_{+, \delta+\lambda}(\varepsilon)} \frac{g_{-, \delta}(\varepsilon)}{g_{-, \delta}(0)}\right]^{-1}\right\} \\
= & \frac{\delta \psi_{\delta+\lambda}^{+}(0) /(\delta+\lambda)+\psi_{\delta}^{-}(0)}{\psi_{\delta+\lambda}^{+}(0)+\psi_{\delta}^{-}(0)} .
\end{aligned}
$$

This completes the proof of Theorem 3.1. 
Taking the limit as $\delta \rightarrow 0+$ in the equality in Theorem 3.1, we obtain the following result.

Corollary 3.1. It holds that

$$
\mathbb{E} \exp \left[-\lambda \int_{0}^{\infty} \mathbf{1}_{\left\{X_{s}<0\right\}} \mathrm{d} s\right]=\frac{\psi_{0}^{-}(0)}{\psi_{\lambda}^{+}(0)+\psi_{0}^{-}(0)},
$$

where $\psi_{0}^{-}(0)$ is specified in (2.7).

Corollary 3.2. In general, we have, for $x>0$,

$$
\mathbb{E}_{x} \exp \left[-\lambda \int_{0}^{e_{\delta}} \mathbf{1}_{\left\{X_{s}<0\right\}} \mathrm{d} s\right]=\frac{g_{-, \delta}(x)}{g_{-, \delta}(0)} \frac{\delta \psi_{\delta+\lambda}^{+}(0) /(\delta+\lambda)+\psi_{\delta}^{-}(0)}{\psi_{\delta+\lambda}^{+}(0)+\psi_{\delta}^{-}(0)}+1-\frac{g_{-, \delta}(x)}{g_{-, \delta}(0)},
$$

and, for $x<0$,

$$
\begin{aligned}
\mathbb{E}_{x} \exp \left[-\lambda \int_{0}^{e_{\delta}} \mathbf{1}_{\left\{X_{s}<0\right\}} \mathrm{d} s\right]= & \frac{g_{+, \delta+\lambda}(x)}{g_{+, \delta+\lambda}(0)} \frac{\delta \psi_{\delta+\lambda}^{+}(0) /(\delta+\lambda)+\psi_{\delta}^{-}(0)}{\psi_{\delta+\lambda}^{+}(0)+\psi_{\delta}^{-}(0)} \\
& +\frac{\delta}{\delta+\lambda}\left(1-\frac{g_{+, \delta+\lambda}(x)}{g_{+, \delta+\lambda}(0)}\right) .
\end{aligned}
$$

Proof. For $x>0$, by comparing $T_{0}$ with $e_{\delta}$ and using the memoryless property, we obtain

$$
\mathbb{E}_{x} \exp \left[-\lambda \int_{0}^{e_{\delta}} \mathbf{1}_{\left\{X_{s}<0\right\}} \mathrm{d} s\right]=\mathbb{P}_{x}\left\{T_{0}<e_{\delta}\right\} \mathbb{E} \exp \left[-\lambda \int_{0}^{e_{\delta}} \mathbf{1}_{\left\{X_{s}<0\right\}} \mathrm{d} s\right]+\mathbb{P}_{x}\left\{e_{\delta}<T_{0}\right\} .
$$

Then (3.3) follows from (2.12) and Theorem 3.1. For $x<0$,

$$
\begin{aligned}
\mathbb{E}_{x} \exp \left[-\lambda \int_{0}^{e_{\delta}} \mathbf{1}_{\left\{X_{s}<0\right\}} \mathrm{d} s\right] \\
=\mathbb{E}_{x}\left[\mathrm{e}^{-\lambda T_{0}} ; T_{0}<e_{\delta}\right] \mathbb{E} \exp \left[-\lambda \int_{0}^{e_{\delta}} \mathbf{1}_{\left\{X_{s}<0\right\}} \mathrm{d} s\right]+\mathbb{E}_{x}\left[\mathrm{e}^{-\lambda e_{\delta}} ; e_{\delta}<T_{0}\right] \\
=\mathbb{E}_{x} \mathrm{e}^{-(\delta+\lambda) T_{0}} \mathbb{E} \exp \left[-\lambda \int_{0}^{e_{\delta}} \mathbf{1}_{\left\{X_{s}<0\right\}} \mathrm{d} s\right]+\frac{\delta}{\delta+\lambda}\left(1-\mathbb{E}_{x} \mathrm{e}^{-(\delta+\lambda) T_{0}}\right) .
\end{aligned}
$$

Then (3.3) also follows from (2.12) and Theorem 3.1.

Using the Laplace transforms on exit times in Section 2, all the results below could be easily extended to a general initial surplus $x \in \mathbb{R}$. However, to keep the paper short, we skip this minor generalization.

The strategy employed in the proof of Theorem 3.1 can be further exploited to find the joint Laplace transforms on the occupation times in multiple regions.

Theorem 3.2. For any $b>0$, we have

$$
\begin{aligned}
\mathbb{E} \exp [- & \left.\lambda_{1} \int_{0}^{e_{\delta}} \mathbf{1}_{\left\{X_{s}<0\right\}} \mathrm{d} s-\lambda_{2} \int_{0}^{e_{\delta}} \mathbf{1}_{\left\{X_{s}>b\right\}} \mathrm{d} s\right] \\
= & {\left[\frac{\delta}{\delta+\lambda_{1}}\left(\frac{f_{2, \delta}(0, b)}{f_{\delta}(0, b)}+\psi_{\delta+\lambda_{2}}^{-}(b)\right) \psi_{\delta+\lambda_{1}}^{+}(0)\right.} \\
& \left.-\left(\frac{\lambda_{2}}{\delta+\lambda_{2}} \frac{f_{2, \delta}(0,0)}{f_{\delta}(0, b)}+\frac{f_{1, \delta}(0, b)}{f_{\delta}(0, b)}\right) \psi_{\delta+\lambda_{2}}^{-}(b)-\frac{f_{12, \delta}(0, b)}{f_{\delta}(0, b)}\right] \\
\times & {\left[\left(\frac{f_{2, \delta}(0, b)}{f_{\delta}(0, b)}+\psi_{\delta+\lambda_{2}}^{-}(b)\right) \psi_{\delta+\lambda_{1}}^{+}(0)-\frac{f_{1, \delta}(0, b)}{f_{\delta}(0, b)} \psi_{\delta+\lambda_{2}}^{-}(b)-\frac{f_{12, \delta}(0, b)}{f_{\delta}(0, b)}\right]^{-1} }
\end{aligned}
$$


and

$$
\begin{aligned}
\mathbb{E}_{b} \exp \left[-\lambda_{1} \int_{0}^{e_{\delta}} \mathbf{1}_{\left\{X_{s}<0\right\}} \mathrm{d} s-\lambda_{2} \int_{0}^{e_{\delta}} \mathbf{1}_{\left\{X_{s}>b\right\}} \mathrm{d} s\right] \\
=\left[\left(\frac{\lambda_{1}}{\delta+\lambda_{1}} \frac{f_{1, \delta}(b, b)}{f_{\delta}(0, b)}+\frac{f_{2, \delta}(0, b)}{f_{\delta}(0, b)}\right) \psi_{\delta+\lambda_{1}}^{+}(0)\right. \\
\left.\quad+\frac{\delta}{\delta+\lambda_{2}}\left(-\frac{f_{1, \delta}(0, b)}{f_{\delta}(0, b)}+\psi_{\delta+\lambda_{1}}^{+}(0)\right) \psi_{\delta+\lambda_{2}}^{-}(b)-\frac{f_{12, \delta}(0, b)}{f_{\delta}(0, b)}\right] \\
\times\left[\left(\frac{f_{2, \delta}(0, b)}{f_{\delta}(0, b)}+\psi_{\delta+\lambda_{2}}^{-}(b)\right) \psi_{\delta+\lambda_{1}}^{+}(0)-\frac{f_{1, \delta}(0, b)}{f_{\delta}(0, b)} \psi_{\delta+\lambda_{2}}^{-}(b)-\frac{f_{12, \delta}(0, b)}{f_{\delta}(0, b)}\right]^{-1} .
\end{aligned}
$$

Proof. Similar to the proof of Theorem 3.1, for arbitrarily small $\varepsilon>0$, we approximate the occupation time $\int_{0}^{e_{\delta}} \mathbf{1}_{\left\{X_{s}<0\right\}} \mathrm{d} s$ by $L_{\varepsilon, 0+}^{\delta}$, the sum of durations up to time $e_{\delta}$ for all the nonoverlapping excursions of the process $X$ that start from 0 and end at $\varepsilon$. Similarly, we approximate $\int_{0}^{e_{\delta}} \mathbf{1}_{\left\{X_{s}>b\right\}} \mathrm{d} s$ by $L_{\varepsilon, b-}^{\delta}$, the sum of durations up to time $e_{\delta}$ for all the excursions of $X$ that start from $b$ and end at $b-\varepsilon$.

For ease of notation, we define $I_{0}(x)=\mathbb{E}_{x} \exp \left[-\lambda_{1} \int_{0}^{e_{\delta}} \mathbf{1}_{\left\{X_{s}<0\right\}} \mathrm{d} s-\lambda_{2} \int_{0}^{e_{\delta}} \mathbf{1}_{\left\{X_{s}>b\right\}} \mathrm{d} s\right]$ and its approximation $I_{\varepsilon}(x)=\mathbb{E}_{x} \exp \left[-\lambda_{1} L_{\varepsilon, 0+}^{\delta}-\lambda_{2} L_{\varepsilon, b-}^{\delta}\right]$ for some $x \in \mathbb{R}$. By the strong Markov property, we have

$$
\begin{aligned}
I_{\varepsilon}(0) & =\mathbb{E}\left[\exp \left[-\lambda_{1} L_{\varepsilon, 0+}^{\delta}-\lambda_{2} L_{\varepsilon, b-}^{\delta}\right] ; T_{\varepsilon}<e_{\delta}\right]+\mathbb{E}\left[\exp \left[-\lambda_{1} L_{\varepsilon, 0+}^{\delta}-\lambda_{2} L_{\varepsilon, b-}^{\delta}\right] ; e_{\delta}<T_{\varepsilon}\right] \\
& =\mathbb{E}\left[\mathrm{e}^{-\lambda_{1} T_{\varepsilon}} ; T_{\varepsilon}<e_{\delta}\right] I_{\varepsilon}(\varepsilon)+\mathbb{E}\left[\mathrm{e}^{-\lambda_{1} e_{\delta}} ; e_{\delta}<T_{\varepsilon}\right] \\
& =\mathbb{E} \mathrm{e}^{-\left(\delta+\lambda_{1}\right) T_{\varepsilon}} I_{\varepsilon}(\varepsilon)+\frac{\delta}{\delta+\lambda_{1}}\left(1-\mathbb{E}^{-\left(\delta+\lambda_{1}\right) T_{\varepsilon}}\right) \\
& =\frac{g_{+, \delta+\lambda_{1}}(0)}{g_{+, \delta+\lambda_{1}}(\varepsilon)} I_{\varepsilon}(\varepsilon)+\frac{\delta}{\delta+\lambda_{1}}\left(1-\frac{g_{+, \delta+\lambda_{1}}(0)}{g_{+, \delta+\lambda_{1}}(\varepsilon)}\right)
\end{aligned}
$$

where the last step is due to (2.12). Furthermore,

$$
\begin{aligned}
I_{\varepsilon}(\varepsilon)= & \mathbb{E}_{\varepsilon}\left[\exp \left[-\lambda_{1} L_{\varepsilon, 0+}^{\delta}-\lambda_{2} L_{\varepsilon, b-}^{\delta}\right] ; T_{0}<T_{b} \wedge e_{\delta}\right] \\
& +\mathbb{E}_{\varepsilon}\left[\exp \left[-\lambda_{1} L_{\varepsilon, 0+}^{\delta}-\lambda_{2} L_{\varepsilon, b-}^{\delta}\right] ; T_{b}<T_{0} \wedge e_{\delta}\right] \\
& +\mathbb{E}_{\varepsilon}\left[\exp \left[-\lambda_{1} L_{\varepsilon, 0+}^{\delta}-\lambda_{2} L_{\varepsilon, b-}^{\delta}\right] ; e_{\delta}<T_{0} \wedge T_{b}\right] \\
= & \mathbb{P}_{\varepsilon}\left\{T_{0}<T_{b} \wedge e_{\delta}\right\} I_{\varepsilon}(0)+\mathbb{P}_{\varepsilon}\left\{T_{b}<T_{0} \wedge e_{\delta}\right\} I_{\varepsilon}(b)+\mathbb{P}_{\varepsilon}\left\{e_{\delta}<T_{0} \wedge T_{b}\right\} \\
= & \mathbb{E}_{\varepsilon}\left[\mathrm{e}^{-\delta T_{0}} ; T_{0}<T_{b}\right] I_{\varepsilon}(0)+\mathbb{E}_{\varepsilon}\left[\mathrm{e}^{-\delta T_{b}} ; T_{b}<T_{0}\right] I_{\varepsilon}(b)+1-\mathbb{E}_{\varepsilon} \mathrm{e}^{-\delta\left(T_{0} \wedge T_{b}\right)} \\
= & \frac{f_{\delta}(\varepsilon, b)}{f_{\delta}(0, b)} I_{\varepsilon}(0)+\frac{f_{\delta}(0, \varepsilon)}{f_{\delta}(0, b)} I_{\varepsilon}(b)+1-\frac{f_{\delta}(0, \varepsilon)+f_{\delta}(\varepsilon, b)}{f_{\delta}(0, b)},
\end{aligned}
$$

where the last step is due to (2.9). Substituting (3.5) into (3.4), solving for $I_{\mathcal{E}}(0)$, and taking the limit, we obtain

$$
\begin{aligned}
& I_{0}(0)=\lim _{\varepsilon \rightarrow 0+} I_{\varepsilon}(0) \\
&=\lim _{\varepsilon \rightarrow 0+}\{[ \frac{\delta}{\delta+\lambda_{1}}\left(1-\frac{g_{+, \delta+\lambda_{1}}(0)}{g_{+, \delta+\lambda_{1}}(\varepsilon)}\right) \\
&\left.+\frac{g_{+, \delta+\lambda_{1}}(0)}{g_{+, \delta+\lambda_{1}}(\varepsilon)}\left(\frac{f_{\delta}(0, \varepsilon)}{f_{\delta}(0, b)} I_{\varepsilon}(b)+1-\frac{f_{\delta}(0, \varepsilon)+f_{\delta}(\varepsilon, b)}{f_{\delta}(0, b)}\right)\right] \\
&\left.\times\left[1-\frac{g_{+, \delta+\lambda_{1}}(0)}{g_{+, \delta+\lambda_{1}}(\varepsilon)} \frac{f_{\delta}(\varepsilon, b)}{f_{\delta}(0, b)}\right]^{-1}\right\}
\end{aligned}
$$




$$
\begin{aligned}
= & {\left[\frac{\delta}{\delta+\lambda_{1}} \psi_{\delta+\lambda_{1}}^{+}(0)+\frac{f_{2, \delta}(0,0)}{f_{\delta}(0, b)} I_{0}(b)-\frac{f_{1, \delta}(0, b)+f_{2, \delta}(0,0)}{f_{\delta}(0, b)}\right] } \\
& \times\left[\psi_{\delta+\lambda_{1}}^{+}(0)-\frac{f_{1, \delta}(0, b)}{f_{\delta}(0, b)}\right]^{-1} .
\end{aligned}
$$

Here, (3.6) is the first equation of the target terms $I_{0}(0)$ and $I_{0}(b)$ we obtained.

Similarly,

$$
\begin{aligned}
I_{\varepsilon}(b)= & \mathbb{E}_{b}\left[\exp \left[-\lambda_{1} L_{\varepsilon, 0+}^{\delta}-\lambda_{2} L_{\varepsilon, b-}^{\delta}\right] ; T_{b-\varepsilon}<e_{\delta}\right] \\
& +\mathbb{E}_{b}\left[\exp \left[-\lambda_{1} L_{\varepsilon, 0+}^{\delta}-\lambda_{2} L_{\varepsilon, b-}^{\delta}\right] ; e_{\delta}<T_{b-\varepsilon}\right] \\
= & \mathbb{E}_{b}\left[\mathrm{e}^{-\lambda_{2} T_{b-\varepsilon}} ; T_{b-\varepsilon}<e_{\delta}\right] I_{\varepsilon}(b-\varepsilon)+\mathbb{E}_{b}\left[\mathrm{e}^{-\lambda_{2} e_{\delta}} ; e_{\delta}<T_{b-\varepsilon}\right] \\
= & \mathbb{E}_{b} \mathrm{e}^{-\left(\delta+\lambda_{2}\right) T_{b-\varepsilon}} I_{\varepsilon}(b-\varepsilon)+\frac{\delta}{\delta+\lambda_{2}}\left(1-\mathbb{E}_{b} \mathrm{e}^{-\left(\delta+\lambda_{2}\right) T_{b-\varepsilon}}\right) \\
= & \frac{g_{-, \delta+\lambda_{2}}(b)}{g_{-, \delta+\lambda_{2}}(b-\varepsilon)} I_{\varepsilon}(b-\varepsilon)+\frac{\delta}{\delta+\lambda_{2}}\left(1-\frac{g_{-, \delta+\lambda_{2}}(b)}{g_{-, \delta+\lambda_{2}}(b-\varepsilon)}\right) .
\end{aligned}
$$

Furthermore,

$$
\begin{aligned}
I_{\varepsilon}(b-\varepsilon)= & \mathbb{E}_{b-\varepsilon}\left[\exp \left[-\lambda_{1} L_{\varepsilon, 0+}^{\delta}-\lambda_{2} L_{\varepsilon, b-}^{\delta}\right] ; T_{b}<T_{0} \wedge e_{\delta}\right] \\
& +\mathbb{E}_{b-\varepsilon}\left[\exp \left[-\lambda_{1} L_{\varepsilon, 0+}^{\delta}-\lambda_{2} L_{\varepsilon, b-}^{\delta}\right] ; T_{0}<T_{b} \wedge e_{\delta}\right] \\
& +\mathbb{E}_{b-\varepsilon}\left[\exp \left[-\lambda_{1} L_{\varepsilon, 0+}^{\delta}-\lambda_{2} L_{\varepsilon, b-}^{\delta}\right] ; e_{\delta}<T_{0} \wedge T_{b}\right] \\
= & \mathbb{P}_{b-\varepsilon}\left\{T_{b}<T_{0} \wedge e_{\delta}\right\} I_{\varepsilon}(b)+\mathbb{P}_{b-\varepsilon}\left\{T_{0}<T_{b} \wedge e_{\delta}\right\} I_{\varepsilon}(0)+\mathbb{P}_{b-\varepsilon}\left\{e_{\delta}<T_{0} \wedge T_{b}\right\} \\
= & \mathbb{E}_{b-\varepsilon}\left[\mathrm{e}^{-\delta T_{b}} ; T_{b}<T_{0}\right] I_{\varepsilon}(b)+\mathbb{E}_{b-\varepsilon}\left[\mathrm{e}^{-\delta T_{0}} ; T_{0}<T_{b}\right] I_{\varepsilon}(0)+1-\mathbb{E}_{b-\varepsilon} \mathrm{e}^{-\delta\left(T_{0} \wedge T_{b}\right)} \\
= & \frac{f_{\delta}(0, b-\varepsilon)}{f_{\delta}(0, b)} I_{\varepsilon}(b)+\frac{f_{\delta}(b-\varepsilon, b)}{f_{\delta}(0, b)} I_{\varepsilon}(0)+1-\frac{f_{\delta}(0, b-\varepsilon)+f_{\delta}(b-\varepsilon, b)}{f_{\delta}(0, b)}
\end{aligned}
$$

Substituting (3.8) into (3.7), solving for $I_{\mathcal{E}}(b)$, and taking the limit, we obtain

$$
\begin{aligned}
I_{0}(b)= & \lim _{\varepsilon \rightarrow 0+} I_{\varepsilon}(b) \\
= & \lim _{\varepsilon \rightarrow 0+}\left\{\left[\frac{\delta}{\delta+\lambda_{2}}\left(1-\frac{g_{-, \delta+\lambda_{2}}(b)}{g_{-, \delta+\lambda_{2}}(b-\varepsilon)}\right)\right.\right. \\
& \left.\quad+\frac{g_{-, \delta+\lambda_{2}}(b)}{g_{-, \delta+\lambda_{2}}(b-\varepsilon)}\left(\frac{f_{\delta}(b-\varepsilon, b)}{f_{\delta}(0, b)} I_{\varepsilon}(0)+1-\frac{f_{\delta}(0, b-\varepsilon)+f_{\delta}(b-\varepsilon, b)}{f_{\delta}(0, b)}\right)\right] \\
& \left.\times\left[1-\frac{g_{-, \delta+\lambda_{2}}(b)}{g_{-, \delta+\lambda_{2}}(b-\varepsilon)} \frac{f_{\delta}(0, b-\varepsilon)}{f_{\delta}(0, b)}\right]^{-1}\right\} \\
= & {\left[\frac{\delta}{\delta+\lambda_{2}} \psi_{\delta+\lambda_{2}}^{-}(b)-\frac{f_{1, \delta}(b, b)}{f_{\delta}(0, b)} I_{0}(0)+\frac{f_{2, \delta}(0, b)+f_{1, \delta}(b, b)}{f_{\delta}(0, b)}\right] } \\
& \times\left[\psi_{\delta+\lambda_{2}}^{-}(b)+\frac{f_{2, \delta}(0, b)}{f_{\delta}(0, b)}\right]^{-1} .
\end{aligned}
$$

Here, (3.9) is the second equation of the target terms $I_{0}(0)$ and $I_{0}(b)$ we obtained. Therefore, solving the linear system composed of (3.6) and (3.9), and simplifying the result using identity (2.13) with $x=0, y=b$, and $r=\delta$, we complete the proof of Theorem 3.2.

Remark 3.1. When $\lambda_{2} \rightarrow 0+$, the first joint Laplace transform in Theorem 3.2 is reduced to Theorem 3.1 using identity (2.14) with $x=0, y=b$, and $r=\delta$. Furthermore, when $\delta \rightarrow 0+$, by (2.5), (2.6), and (2.8), both of the Laplace transforms in Theorem 3.2 are equal to 0 . 
Let $b \rightarrow 0+$ in Theorem 3.2, and by the facts that $f_{12, \delta}(0,0)=0$ and $f_{1, \delta}(0,0)=$ $-f_{2, \delta}(0,0)$, we obtain the following compact expression.

Corollary 3.3. It holds that

$$
\begin{aligned}
\mathbb{E} \exp \left[-\lambda_{1} \int_{0}^{e_{\delta}} \mathbf{1}_{\left\{X_{s}<0\right\}} \mathrm{d} s-\lambda_{2} \int_{0}^{e_{\delta}} \mathbf{1}_{\left\{X_{s}>0\right\}} \mathrm{d} s\right] \\
=\frac{\delta \psi_{\delta+\lambda_{1}}^{+}(0) /\left(\delta+\lambda_{1}\right)+\delta \psi_{\delta+\lambda_{2}}^{-}(0) /\left(\delta+\lambda_{2}\right)}{\psi_{\delta+\lambda_{1}}^{+}(0)+\psi_{\delta+\lambda_{2}}^{-}(0)} .
\end{aligned}
$$

By (2.11), letting $\lambda_{2} \rightarrow \infty$ and $\lambda_{1} \rightarrow \infty$ in the first and second equations of Theorem 3.2, respectively, we obtain the following result.

Corollary 3.4. For any $a<0$, we have

$$
\mathbb{E}\left[\exp \left[-\lambda \int_{0}^{e_{\delta}} \mathbf{1}_{\left\{X_{s}<0\right\}} \mathrm{d} s\right] ; e_{\delta}<T_{b}\right]=\frac{\delta f_{\delta}(0, b) \psi_{\delta+\lambda}^{+}(0) /(\delta+\lambda)-f_{2, \delta}(0,0)-f_{1, \delta}(0, b)}{f_{\delta}(0, b) \psi_{\delta+\lambda}^{+}(0)-f_{1, \delta}(0, b)}
$$

and

$$
\begin{aligned}
& \mathbb{E}_{b}\left[\exp \left[-\lambda \int_{0}^{e_{\delta}} \mathbf{1}_{\left\{X_{s}>b\right\}} \mathrm{d} s\right] ; e_{\delta}<T_{0}\right] \\
& =\frac{\delta f_{\delta}(0, b) \psi_{\delta+\lambda}^{-}(b) /(\delta+\lambda)+f_{1, \delta}(b, b)+f_{2, \delta}(0, b)}{f_{\delta}(0, b) \psi_{\delta+\lambda}^{-}(b)+f_{2, \delta}(0, b)} .
\end{aligned}
$$

Next we consider the joint Laplace transforms on the occupation times in a bounded region and an unbounded region.

Theorem 3.3. For any $a<0$, we have

$$
\begin{aligned}
\mathbb{E} \exp [- & \left.\lambda_{1} \int_{0}^{e_{\delta}} \mathbf{1}_{\left\{a \leq X_{s}<0\right\}} \mathrm{d} s-\lambda_{2} \int_{0}^{e_{\delta}} \mathbf{1}_{\left\{X_{s}<a\right\}} \mathrm{d} s\right] \\
= & {\left[\frac{\delta\left(\delta+\lambda_{2}\right) f_{2, \delta+\lambda_{1}}(a, 0)+\delta\left(\lambda_{1}-\lambda_{2}\right) f_{2, \delta+\lambda_{1}}(0,0)}{\left(\delta+\lambda_{1}\right)\left(\delta+\lambda_{2}\right) f_{\delta+\lambda_{1}}(a, 0)} \psi_{\delta+\lambda_{2}}^{+}(a)-\frac{f_{1, \delta+\lambda_{1}}(a, 0)}{f_{\delta+\lambda_{1}}(a, 0)} \psi_{\delta}^{-}(0)\right.} \\
& \left.+\psi_{\delta+\lambda_{2}}^{+}(a) \psi_{\delta}^{-}(0)-\frac{\delta f_{12, \delta+\lambda_{1}}(a, 0)}{\left(\delta+\lambda_{1}\right) f_{\delta+\lambda_{1}}(a, 0)}\right] \\
& \times\left[\frac{f_{2, \delta+\lambda_{1}}(a, 0)}{f_{\delta+\lambda_{1}}(a, 0)} \psi_{\delta+\lambda_{2}}^{+}(a)-\frac{f_{1, \delta+\lambda_{1}}(a, 0)}{f_{\delta+\lambda_{1}}(a, 0)} \psi_{\delta}^{-}(0)+\psi_{\delta+\lambda_{2}}^{+}(a) \psi_{\delta}^{-}(0)\right. \\
& \left.\quad-\frac{f_{12, \delta+\lambda_{1}}(a, 0)}{f_{\delta+\lambda_{1}}(a, 0)}\right]^{-1}
\end{aligned}
$$

and

$$
\begin{aligned}
\mathbb{E}_{a} \exp [ & \left.-\lambda_{1} \int_{0}^{e_{\delta}} \mathbf{1}_{\left\{a \leq X_{s}<0\right\}} \mathrm{d} s-\lambda_{2} \int_{0}^{e_{\delta}} \mathbf{1}_{\left\{X_{s}<a\right\}} \mathrm{d} s\right] \\
= & {\left[\frac{\delta f_{2, \delta+\lambda_{1}}(a, 0)}{\left(\delta+\lambda_{2}\right) f_{\delta+\lambda_{1}}(a, 0)} \psi_{\delta+\lambda_{2}}^{+}(a)-\frac{\delta f_{1, \delta+\lambda_{1}}(a, 0)-\lambda_{1} f_{2, \delta+\lambda_{1}}(a, a)}{\left(\delta+\lambda_{1}\right) f_{\delta+\lambda_{1}}(a, 0)} \psi_{\delta}^{-}(0)\right.} \\
& \left.+\frac{\delta}{\delta+\lambda_{2}} \psi_{\delta+\lambda_{2}}^{+}(a) \psi_{\delta}^{-}(0)-\frac{\delta f_{12, \delta+\lambda_{1}}(a, 0)}{\left(\delta+\lambda_{1}\right) f_{\delta+\lambda_{1}}(a, 0)}\right] \\
\times & {\left[\frac{f_{2, \delta+\lambda_{1}}(a, 0)}{f_{\delta+\lambda_{1}}(a, 0)} \psi_{\delta+\lambda_{2}}^{+}(a)-\frac{f_{1, \delta+\lambda_{1}}(a, 0)}{f_{\delta+\lambda_{1}}(a, 0)} \psi_{\delta}^{-}(0)+\psi_{\delta+\lambda_{2}}^{+}(a) \psi_{\delta}^{-}(0)\right.} \\
& \left.\quad-\frac{f_{12, \delta+\lambda_{1}}(a, 0)}{f_{\delta+\lambda_{1}}(a, 0)}\right]^{-1} .
\end{aligned}
$$


Proof. For arbitrarily small $\varepsilon>0$, we approximate the occupation time $\int_{0}^{e_{\delta}} \mathbf{1}_{\left\{a \leq X_{s}<0\right\}} \mathrm{d} s$ by $L_{\varepsilon, a, 0}^{\delta}$, the sum of durations up to time $e_{\delta}$ for all the excursions of $X$ that either start from 0 and end at $\varepsilon$, avoiding $a$, or start from 0 and end at $a$, avoiding $\varepsilon$. Similarly, we approximate $\int_{0}^{e_{\delta}} \mathbf{1}_{\left\{X_{s}<a\right\}} \mathrm{d} s$ by $L_{\varepsilon, a+}^{\delta}$, the sum of durations up to time $e_{\delta}$ for all the excursions of $X$ that start from $a$ and end at $a+\varepsilon$.

We define $J_{0}(x)=\mathbb{E}_{x} \exp \left[-\lambda_{1} \int_{0}^{e_{\delta}} \mathbf{1}_{\left\{a \leq X_{s}<0\right\}} \mathrm{d} s-\lambda_{2} \int_{0}^{e_{\delta}} \mathbf{1}_{\left\{X_{s}<a\right\}} \mathrm{d} s\right]$ and its approximation $J_{\varepsilon}(x)=\mathbb{E}_{x} \exp \left[-\lambda_{1} L_{\varepsilon, a, 0}^{\delta}-\lambda_{2} L_{\varepsilon, a+}^{\delta}\right]$ for some $x \in \mathbb{R}$. Then we have

$$
\begin{aligned}
J_{\varepsilon}(0)= & \mathbb{E}\left[\exp \left[-\lambda_{1} L_{\varepsilon, a, 0}^{\delta}-\lambda_{2} L_{\varepsilon, a+}^{\delta}\right] ; T_{\varepsilon}<T_{a} \wedge e_{\delta}\right] \\
& +\mathbb{E}\left[\exp \left[-\lambda_{1} L_{\varepsilon, a, 0}^{\delta}-\lambda_{2} L_{\varepsilon, a+}^{\delta}\right] ; T_{a}<T_{\varepsilon} \wedge e_{\delta}\right] \\
& +\mathbb{E}\left[\exp \left[-\lambda_{1} L_{\varepsilon, a, 0}^{\delta}-\lambda_{2} L_{\varepsilon, a+}^{\delta}\right] ; e_{\delta}<T_{\varepsilon} \wedge T_{a}\right] \\
= & \mathbb{E}\left[\mathrm{e}^{-\lambda_{1} T_{\varepsilon}} ; T_{\varepsilon}<T_{a} \wedge e_{\delta}\right] J_{\varepsilon}(\varepsilon)+\mathbb{E}\left[\mathrm{e}^{-\lambda_{1} T_{a}} ; T_{a}<T_{\varepsilon} \wedge e_{\delta}\right] J_{\varepsilon}(a) \\
& +\mathbb{E}\left[\mathrm{e}^{-\lambda_{1} e_{\delta}} ; e_{\delta}<T_{\varepsilon} \wedge T_{a}\right] \\
= & \mathbb{E}\left[\mathrm{e}^{-\left(\delta+\lambda_{1}\right) T_{\varepsilon}} ; T_{\varepsilon}<T_{a}\right] J_{\varepsilon}(\varepsilon)+\mathbb{E}\left[\mathrm{e}^{-\left(\delta+\lambda_{1}\right) T_{a}} ; T_{a}<T_{\varepsilon}\right] J_{\varepsilon}(a) \\
& +\frac{\delta\left(1-\mathbb{E} \mathrm{e}^{-\left(\delta+\lambda_{1}\right)\left(T_{\varepsilon} \wedge T_{a}\right)}\right)}{\delta+\lambda_{1}} \\
& \frac{f_{\delta+\lambda_{1}}(a, 0)}{f_{\delta+\lambda_{1}}(a, \varepsilon)} J_{\varepsilon}(\varepsilon)+\frac{f_{\delta+\lambda_{1}}(0, \varepsilon)}{f_{\delta+\lambda_{1}}(a, \varepsilon)} J_{\varepsilon}(a)+\frac{\delta}{\delta+\lambda_{1}}\left(1-\frac{f_{\delta+\lambda_{1}}(a, 0)+f_{\delta+\lambda_{1}}(0, \varepsilon)}{f_{\delta+\lambda_{1}}(a, \varepsilon)}\right) .
\end{aligned}
$$

Furthermore,

$$
\begin{aligned}
J_{\varepsilon}(\varepsilon) & =\mathbb{P}_{\varepsilon}\left\{T_{0}<e_{\delta}\right\} J_{\varepsilon}(0)+\mathbb{P}_{\varepsilon}\left\{e_{\delta}<T_{0}\right\} \\
& =\mathbb{E}_{\varepsilon} \mathrm{e}^{-\delta T_{0}} J_{\varepsilon}(0)+1-\mathbb{E}_{\varepsilon} \mathrm{e}^{-\delta T_{0}} \\
& =\frac{g_{-, \delta}(\varepsilon)}{g_{-, \delta}(0)} J_{\varepsilon}(0)+1-\frac{g_{-, \delta}(\varepsilon)}{g_{-, \delta}(0)}
\end{aligned}
$$

where the last step is due to (2.12). Substituting (3.11) into (3.10), solving for $J_{\varepsilon}(0)$, and taking the limit, we obtain

$$
\begin{aligned}
& J_{0}(0)= \lim _{\varepsilon \rightarrow 0+} J_{\varepsilon}(0) \\
&=\lim _{\varepsilon \rightarrow 0+}\left\{\left[\frac{f_{\delta+\lambda_{1}}(a, 0)}{f_{\delta+\lambda_{1}}(a, \varepsilon)}\left(1-\frac{g_{-, \delta}(\varepsilon)}{g_{-, \delta}(0)}\right)+\frac{f_{\delta+\lambda_{1}}(0, \varepsilon)}{f_{\delta+\lambda_{1}}(a, \varepsilon)} J_{\varepsilon}(a)\right.\right. \\
&\left.\quad+\frac{\delta}{\delta+\lambda_{1}}\left(1-\frac{f_{\delta+\lambda_{1}}(a, 0)+f_{\delta+\lambda_{1}}(0, \varepsilon)}{f_{\delta+\lambda_{1}}(a, \varepsilon)}\right)\right] \\
&\left.\times\left[1-\frac{f_{\delta+\lambda_{1}}(a, 0)}{f_{\delta+\lambda_{1}}(a, \varepsilon)} \frac{g_{-, \delta}(\varepsilon)}{g_{-, \delta}(0)}\right]^{-1}\right\} \\
&= {\left[\psi_{\delta}^{-}(0)+\frac{f_{2, \delta+\lambda_{1}}(0,0)}{f_{\delta+\lambda_{1}}(a, 0)} J_{0}(a)+\frac{\delta}{\delta+\lambda_{1}} \frac{f_{2, \delta+\lambda_{1}}(a, 0)-f_{2, \delta+\lambda_{1}}(0,0)}{f_{\delta+\lambda_{1}}(a, 0)}\right] } \\
& \times\left[\psi_{\delta}^{-}(0)+\frac{f_{2, \delta+\lambda_{1}}(a, 0)}{f_{\delta+\lambda_{1}}(a, 0)}\right]^{-1} .
\end{aligned}
$$

Here, (3.12) is the first equation of the target terms $J_{0}(0)$ and $J_{0}(a)$ we obtained. 
Similarly,

$$
\begin{aligned}
J_{\varepsilon}(a)= & \mathbb{E}_{a}\left[\exp \left[-\lambda_{1} L_{\varepsilon, a, 0}^{\delta}-\lambda_{2} L_{\varepsilon, a+}^{\delta}\right] ; T_{a+\varepsilon}<e_{\delta}\right] \\
& +\mathbb{E}_{a}\left[\exp \left[-\lambda_{1} L_{\varepsilon, a, 0}^{\delta}-\lambda_{2} L_{\varepsilon, a+}^{\delta}\right] ; e_{\delta}<T_{a+\varepsilon}\right] \\
= & \mathbb{E}_{a}\left[\mathrm{e}^{-\lambda_{2} T_{a+\varepsilon}} ; T_{a+\varepsilon}<e_{\delta}\right] J_{\varepsilon}(a+\varepsilon)+\mathbb{E}_{a}\left[\mathrm{e}^{-\lambda_{2} e_{\delta}} ; e_{\delta}<T_{a+\varepsilon}\right] \\
= & \mathbb{E}_{a} \mathrm{e}^{-\left(\delta+\lambda_{2}\right) T_{a+\varepsilon}} J_{\varepsilon}(a+\varepsilon)+\frac{\delta}{\delta+\lambda_{2}}\left(1-\mathbb{E}_{a} \mathrm{e}^{\left.-\left(\delta+\lambda_{2}\right) T_{a+\varepsilon}\right)}\right. \\
= & \frac{g_{+, \delta+\lambda_{2}}(a)}{g_{+, \delta+\lambda_{2}}(a+\varepsilon)} J_{\varepsilon}(a+\varepsilon)+\frac{\delta}{\delta+\lambda_{2}}\left(1-\frac{g_{+, \delta+\lambda_{2}}(a)}{g_{+, \delta+\lambda_{2}}(a+\varepsilon)}\right) .
\end{aligned}
$$

Furthermore,

$$
\begin{aligned}
J_{\varepsilon}(a+\varepsilon)= & \mathbb{E}_{a+\varepsilon}\left[\exp \left[-\lambda_{1} L_{\varepsilon, a, 0}^{\delta}-\lambda_{2} L_{\varepsilon, a+}^{\delta}\right] ; T_{a}<T_{0} \wedge e_{\delta}\right] \\
& +\mathbb{E}_{a+\varepsilon}\left[\exp \left[-\lambda_{1} L_{\varepsilon, a, 0}^{\delta}-\lambda_{2} L_{\varepsilon, a+}^{\delta}\right] ; T_{0}<T_{a} \wedge e_{\delta}\right] \\
& +\mathbb{E}_{a+\varepsilon}\left[\exp \left[-\lambda_{1} L_{\varepsilon, a, 0}^{\delta}-\lambda_{2} L_{\varepsilon, a+}^{\delta}\right] ; e_{\delta}<T_{a} \wedge T_{0}\right] \\
= & \mathbb{E}_{a+\varepsilon}\left[\mathrm{e}^{-\lambda_{1} T_{a}} ; T_{a}<T_{0} \wedge e_{\delta}\right] J_{\varepsilon}(a)+\mathbb{E}_{a+\varepsilon}\left[\mathrm{e}^{-\lambda_{1} T_{0}} ; T_{0}<T_{a} \wedge e_{\delta}\right] J_{\varepsilon}(0) \\
& +\mathbb{E}_{a+\varepsilon}\left[\mathrm{e}^{-\lambda_{1} e_{\delta}} ; e_{\delta}<T_{a} \wedge T_{0}\right] \\
= & \mathbb{E}_{a+\varepsilon}\left[\mathrm{e}^{-\left(\delta+\lambda_{1}\right) T_{a}} ; T_{a}<T_{0}\right] J_{\varepsilon}(a)+\mathbb{E}_{a+\varepsilon}\left[\mathrm{e}^{-\left(\delta+\lambda_{1}\right) T_{0}} ; T_{0}<T_{a}\right] J_{\varepsilon}(0) \\
& +\frac{\delta}{\delta+\lambda_{1}}\left(1-\mathbb{E}_{a+\varepsilon}\left[\mathrm{e}^{-\left(\delta+\lambda_{1}\right)\left(T_{a} \wedge T_{0}\right)}\right]\right) \\
= & \frac{f_{\delta+\lambda_{1}}(a+\varepsilon, 0)}{f_{\delta+\lambda_{1}}(a, 0)} J_{\varepsilon}(a)+\frac{f_{\delta+\lambda_{1}}(a, a+\varepsilon)}{f_{\delta+\lambda_{1}}(a, 0)} J_{\varepsilon}(0) \\
& +\frac{\delta}{\delta+\lambda_{1}}\left(1-\frac{f_{\delta+\lambda_{1}}(a, a+\varepsilon)+f_{\delta+\lambda_{1}}(a+\varepsilon, 0)}{f_{\delta+\lambda_{1}}(a, 0)}\right)
\end{aligned}
$$

Substituting (3.14) into (3.13), solving for $J_{\varepsilon}(a)$, and taking the limit, we obtain

$$
\begin{aligned}
J_{0}(a)= & \lim _{\varepsilon \rightarrow 0+} J_{\varepsilon}(a) \\
= & \lim _{\varepsilon \rightarrow 0+}\left\{\left[\frac{g_{+, \delta+\lambda_{2}}(a)}{g_{+, \delta+\lambda_{2}}(a+\varepsilon)} \frac{\delta}{\delta+\lambda_{1}}\left(1-\frac{f_{\delta+\lambda_{1}}(a, a+\varepsilon)+f_{\delta+\lambda_{1}}(a+\varepsilon, 0)}{f_{\delta+\lambda_{1}}(a, 0)}\right)\right.\right. \\
& \left.+\frac{\delta}{\delta+\lambda_{2}}\left(1-\frac{g_{+, \delta+\lambda_{2}}(a)}{g_{+, \delta+\lambda_{2}}(a+\varepsilon)}\right)\right] \\
& \left.\times\left[1-\frac{g_{+, \delta+\lambda_{2}}(a)}{g_{+, \delta+\lambda_{2}}(a+\varepsilon)} \frac{f_{\delta+\lambda_{1}}(a+\varepsilon, 0)}{f_{\delta+\lambda_{1}}(a, 0)}\right]^{-1}\right\} \\
& +\lim _{\varepsilon \rightarrow 0+}\left\{J_{\varepsilon}(0)\left[\frac{g_{+, \delta+\lambda_{2}}(a)}{g_{+, \delta+\lambda_{2}}(a+\varepsilon)} \frac{f_{\delta+\lambda_{1}}(a, a+\varepsilon)}{f_{\delta+\lambda_{1}}(a, 0)}\right]\right. \\
= & \left.\left.\times \frac{\delta}{\delta+\lambda_{1}} \frac{-f_{1, \delta+\lambda_{1}}(a, 0)-f_{2, \delta+\lambda_{1}}(a, a)}{g_{\delta+, \delta+\lambda_{1}}(a, 0)}+\frac{f_{\delta+\lambda_{1}}(a+\varepsilon, 0)}{f_{\delta+\lambda_{1}}(a, 0)}\right]^{-1}\right\} \\
& \left.\times\left[\psi_{\delta+\lambda_{2}}^{+}(a)-\frac{f_{1, \delta+\lambda_{1}}(a, 0)}{f_{\delta+\lambda_{1}}(a, 0)}\right]_{\delta+\lambda_{2}}^{-1}(a)+\frac{f_{2, \delta+\lambda_{1}}(a, a)}{f_{\delta+\lambda_{1}}(a, 0)} J_{0}(0)\right]
\end{aligned}
$$


Here, (3.15) is the second equation of the target terms $J_{0}(0)$ and $J_{0}(a)$ we obtained. Finally, solving the linear system composed of (3.12) and (3.15), and simplifying the result using identity (2.13) with $x=a, y=0$, and $r=\delta+\lambda_{1}$, we complete the proof of Theorem 3.3.

Remark 3.2. When $\lambda_{1}=\lambda_{2}=\lambda$, the first joint Laplace transform in Theorem 3.3 is reduced to Theorem 3.1 using identity (2.15) with $x=a, y=0$, and $r=\delta+\lambda$. Furthermore, when $\lambda_{1} \rightarrow 0+, \lambda_{2}=\lambda$, and $a=0$, the second joint Laplace transform in Theorem 3.3 is reduced to Theorem 3.1 using identity (2.14) with $x=a, y=0$, and $r=\delta$.

Letting $\delta \rightarrow 0+$ in Theorem 3.3, we obtain the following corollary.

Corollary 3.5. For any $a<0$, we have

$$
\begin{aligned}
\mathbb{E} \exp \left[-\lambda_{1} \int_{0}^{\infty} \mathbf{1}_{\left\{a \leq X_{s}<0\right\}} \mathrm{d} s-\lambda_{2} \int_{0}^{\infty} \mathbf{1}_{\left\{X_{s}<a\right\}} \mathrm{d} s\right] \\
=\frac{-f_{1, \lambda_{1}}(a, 0) \psi_{0}^{-}(0)+f_{\lambda_{1}}(a, 0) \psi_{\lambda_{2}}^{+}(a) \psi_{0}^{-}(0)}{f_{2, \lambda_{1}}(a, 0) \psi_{\lambda_{2}}^{+}(a)-f_{1, \lambda_{1}}(a, 0) \psi_{0}^{-}(0)+f_{\lambda_{1}}(a, 0) \psi_{\lambda_{2}}^{+}(a) \psi_{0}^{-}(0)-f_{12, \lambda_{1}}(a, 0)}
\end{aligned}
$$

and

$$
\begin{aligned}
\mathbb{E}_{a} \exp \left[-\lambda_{1} \int_{0}^{\infty} \mathbf{1}_{\left\{a \leq X_{s}<0\right\}} \mathrm{d} s-\lambda_{2} \int_{0}^{\infty} \mathbf{1}_{\left\{X_{s}<a\right\}} \mathrm{d} s\right] \\
=\frac{f_{2, \delta+\lambda_{1}}(a, a) \psi_{0}^{-}(0)}{f_{2, \lambda_{1}}(a, 0) \psi_{\lambda_{2}}^{+}(a)-f_{1, \lambda_{1}}(a, 0) \psi_{0}^{-}(0)+f_{\lambda_{1}}(a, 0) \psi_{\lambda_{2}}^{+}(a) \psi_{0}^{-}(0)-f_{12, \lambda_{1}}(a, 0)} .
\end{aligned}
$$

Letting $\lambda_{2} \rightarrow 0+$ in Theorem 3.3, and using the identity $f_{2, \delta+\lambda}(a, a)=-f_{1, \delta+\lambda}(a, a)$, we obtain the following result.

Corollary 3.6. For any $a<0$, we have

$$
\begin{aligned}
\mathbb{E} \exp [- & \left.\lambda \int_{0}^{e_{\delta}} \mathbf{1}_{\left\{a \leq X_{s}<0\right\}} \mathrm{d} s\right] \\
= & {\left[\frac{\delta f_{2, \delta+\lambda}(a, 0)+\lambda f_{2, \delta+\lambda}(0,0)}{(\delta+\lambda) f_{\delta+\lambda}(a, 0)} \psi_{\delta}^{+}(a)-\frac{f_{1, \delta+\lambda}(a, 0)}{f_{\delta+\lambda}(a, 0)} \psi_{\delta}^{-}(0)+\psi_{\delta}^{+}(a) \psi_{\delta}^{-}(0)\right.} \\
& \left.-\frac{\delta f_{12, \delta+\lambda}(a, 0)}{(\delta+\lambda) f_{\delta+\lambda}(a, 0)}\right] \\
& \times\left[\frac{f_{2, \delta+\lambda}(a, 0)}{f_{\delta+\lambda}(a, 0)} \psi_{\delta}^{+}(a)-\frac{f_{1, \delta+\lambda}(a, 0)}{f_{\delta+\lambda}(a, 0)} \psi_{\delta}^{-}(0)+\psi_{\delta}^{+}(a) \psi_{\delta}^{-}(0)-\frac{f_{12, \delta+\lambda}(a, 0)}{f_{\delta+\lambda}(a, 0)}\right]^{-1}
\end{aligned}
$$

and

$$
\begin{aligned}
\mathbb{E}_{a} \exp [ & \left.-\lambda \int_{0}^{e_{\delta}} \mathbf{1}_{\left\{a \leq X_{s}<0\right\}} \mathrm{d} s\right] \\
= & {\left[\frac{f_{2, \delta+\lambda}(a, 0)}{f_{\delta+\lambda}(a, 0)} \psi_{\delta}^{+}(a)-\frac{\delta f_{1, \delta+\lambda}(a, 0)+\lambda f_{1, \delta+\lambda}(a, a)}{(\delta+\lambda) f_{\delta+\lambda}(a, 0)} \psi_{\delta}^{-}(0)+\psi_{\delta}^{+}(a) \psi_{\delta}^{-}(0)\right.} \\
& \left.-\frac{\delta f_{12, \delta+\lambda}(a, 0)}{(\delta+\lambda) f_{\delta+\lambda}(a, 0)}\right] \\
& \times\left[\frac{f_{2, \delta+\lambda}(a, 0)}{f_{\delta+\lambda}(a, 0)} \psi_{\delta}^{+}(a)-\frac{f_{1, \delta+\lambda}(a, 0)}{f_{\delta+\lambda}(a, 0)} \psi_{\delta}^{-}(0)+\psi_{\delta}^{+}(a) \psi_{\delta}^{-}(0)-\frac{f_{12, \delta+\lambda}(a, 0)}{f_{\delta+\lambda}(a, 0)}\right]^{-1} .
\end{aligned}
$$


Letting $\delta \rightarrow 0+$ in Corollary 3.6, we obtain the following simplified expression.

Corollary 3.7. For any $a<0$, we have

$$
\begin{aligned}
\mathbb{E} \exp \left[-\lambda \int_{0}^{\infty} \mathbf{1}_{\left\{a \leq X_{s}<0\right\}} \mathrm{d} s\right] \\
=\frac{f_{2, \lambda}(0,0) \psi_{0}^{+}(a)-f_{1, \lambda}(a, 0) \psi_{0}^{-}(0)+f_{\lambda}(a, 0) \psi_{0}^{+}(a) \psi_{0}^{-}(0)}{f_{2, \lambda}(a, 0) \psi_{0}^{+}(a)-f_{1, \lambda}(a, 0) \psi_{0}^{-}(0)+f_{\lambda}(a, 0) \psi_{0}^{+}(a) \psi_{0}^{-}(0)-f_{12, \lambda}(a, 0)}
\end{aligned}
$$

and

$$
\begin{aligned}
\mathbb{E}_{a} \exp \left[-\lambda \int_{0}^{\infty} \mathbf{1}_{\left\{a \leq X_{s}<0\right\}} \mathrm{d} s\right] \\
\quad=\frac{f_{2, \lambda}(a, 0) \psi_{0}^{+}(a)-f_{1, \lambda}(a, a) \psi_{0}^{-}(0)+f_{\lambda}(a, 0) \psi_{0}^{+}(a) \psi_{0}^{-}(0)}{f_{2, \lambda}(a, 0) \psi_{0}^{+}(a)-f_{1, \lambda}(a, 0) \psi_{0}^{-}(0)+f_{\lambda}(a, 0) \psi_{0}^{+}(a) \psi_{0}^{-}(0)-f_{12, \lambda}(a, 0)} .
\end{aligned}
$$

Remark 3.3. As $a \rightarrow-\infty$, by (2.10), the first Laplace transforms in Corollaries 3.6 and 3.7 are reduced to Theorem 3.1 and Corollary 3.1, respectively.

Letting $\lambda_{2} \rightarrow \infty$ in the first equation of Theorem 3.3, by (2.11), we obtain the following result.

Corollary 3.8. For any $a<0$, we have

$$
\begin{aligned}
\mathbb{E}\left[\exp \left[-\lambda \int_{0}^{e_{\delta}} \mathbf{1}_{\left\{a \leq X_{s}<0\right\}} \mathrm{d} s\right] ; e_{\delta}<T_{a}\right] \\
=\frac{\delta\left(f_{2, \delta+\lambda}(a, 0)-f_{2, \delta+\lambda}(0,0)\right) /(\delta+\lambda)+f_{\delta+\lambda}(a, 0) \psi_{\delta}^{-}(0)}{f_{2, \delta+\lambda}(a, 0)+f_{\delta+\lambda}(a, 0) \psi_{\delta}^{-}(0)} .
\end{aligned}
$$

\section{Some applications to diffusion risk processes}

The Laplace transforms in Section 3 are motivated by the study of two classes of risk models that we are going to introduce below.

\subsection{The risk process with random observations}

Let the surplus process $X$ in a risk model be the time-homogeneous diffusion process defined in (2.1) with initial value $x_{0}>0$, and let $N$ be an independent Poisson process with constant rate $\lambda>0$ of consecutive arrival times $0<\tau_{1}<\tau_{2}<\cdots$. Suppose that the values of the process $X$ are only observed at the times $\left(\tau_{i}\right)$. The ruin time for this model is defined as

$$
\tau_{\lambda}=\inf _{i \geq 1}\left\{\tau_{i}: X_{\tau_{i}}<0\right\},
$$

with the convention that inf $\varnothing=\infty$.

There is a natural connection between the survival probability for this risk process and the occupation time for the underlying process $X$. Actually, for any $t>0$,

$$
\begin{aligned}
\mathbb{P}_{x_{0}}\left\{\tau_{\lambda}>t\right\} & =\mathbb{E}_{x_{0}} \mathbb{P}\left\{\left\{\tau_{i}\right\} \cap\left\{s \leq t: X_{s}<0\right\}=\varnothing \mid X\right\} \\
& =\mathbb{E}_{x_{0}} \mathbb{E}\left[\exp \left[-\lambda \int_{0}^{t} \mathbf{1}_{\left\{X_{s}<0\right\}} \mathrm{d} s\right] \mid X\right] \\
& =\mathbb{E}_{x_{0}} \exp \left[-\lambda \int_{0}^{t} \mathbf{1}_{\left\{X_{s}<0\right\}} \mathrm{d} s\right] .
\end{aligned}
$$


Therefore, the Laplace transform for the ruin time $\tau_{\lambda}$ is

$$
\mathbb{E}_{x_{0}} \mathrm{e}^{-\delta \tau_{\lambda}}=\mathbb{P}_{x_{0}}\left\{\tau_{\lambda}<e_{\delta}\right\}=1-\mathbb{E}_{x_{0}} \exp \left[-\lambda \int_{0}^{e_{\delta}} \mathbf{1}_{\left\{X_{s}<0\right\}} \mathrm{d} s\right]
$$

Furthermore, by Corollary 3.2,

$$
\mathbb{E}_{x_{0}} \mathrm{e}^{-\delta \tau_{\lambda}}=\frac{\lambda}{\delta+\lambda} \frac{g_{-, \delta}\left(x_{0}\right)}{g_{-, \delta}(0)} \frac{\psi_{\delta+\lambda}^{+}(0)}{\psi_{\delta+\lambda}^{+}(0)+\psi_{\delta}^{-}(0)} .
$$

Note that, when $\lambda \rightarrow \infty$ and $\delta \rightarrow 0+$, (4.1) is reduced to the usual ruin probability by (2.5) and (2.11).

Moreover, for $b>0$, write

$$
\tau_{\lambda, b}=\inf _{i \geq 1}\left\{\tau_{i}: X_{\tau_{i}}<0 \text { or } X_{\tau_{i}}>b\right\}
$$

for the 'two-sided exit time' of this model. Then, by conditioning, its Laplace transform is given by

$$
\mathbb{E}_{x_{0}} \mathrm{e}^{-\delta \tau_{\lambda, b}}=\mathbb{P}_{x_{0}}\left\{\tau_{\lambda, b}<e_{\delta}\right\}=1-\mathbb{E}_{x_{0}} \exp \left[-\lambda \int_{0}^{e_{\delta}} \mathbf{1}_{\left\{X_{s}<0\right\}} \mathrm{d} s-\lambda \int_{0}^{e_{\delta}} \mathbf{1}_{\left\{X_{s}>b\right\}} \mathrm{d} s\right],
$$

which can be found by Theorem 3.2.

Fixing a level $a \leq 0$ and a positive integer $m$, for the abovementioned diffusion risk model with exponential interobservation times, we can further propose another generalized ruin as the event that either there is at least one observation of $X$ below level $a$ or there are at least $m$ observations of $X$ between level $a$ and level 0 . Note that such a ruin has the spirit of a new ruin model subject to Chapters 7 and 11 of the bankruptcy code which was first introduced in [16]. Write $\tau_{\lambda}^{m, a}$ for the first Poisson arrival time $\tau_{i}$ when such a ruin occurs. By definition, $\tau_{\lambda}^{1,-\infty}=\tau_{\lambda}$.

Conditioning on $X$ and using the properties for Poisson processes, for any $t>0$, we obtain

$$
\begin{aligned}
\mathbb{P}_{x_{0}}\left\{\tau_{\lambda}^{m, a}>t\right\} & \\
& =\sum_{i=0}^{m-1} \mathbb{E}_{x_{0}}\left[\frac{\left(\lambda \int_{0}^{t} \mathbf{1}_{\left\{a \leq X_{s}<0\right\}} \mathrm{d} s\right)^{i}}{i !} \exp \left[-\lambda \int_{0}^{t} \mathbf{1}_{\left\{a \leq X_{s}<0\right\}} \mathrm{d} s\right] \exp \left[-\lambda \int_{0}^{t} \mathbf{1}_{\left\{X_{s}<a\right\}} \mathrm{d} s\right]\right] \\
& =\sum_{i=0}^{m-1} \mathbb{E}_{x_{0}}\left[\frac{\left(\lambda \int_{0}^{t} \mathbf{1}_{\left\{a \leq X_{s}<0\right\}} \mathrm{d} s\right)^{i}}{i !} \exp \left[-\lambda \int_{0}^{t} \mathbf{1}_{\left\{X_{s}<0\right\}} \mathrm{d} s\right]\right] .
\end{aligned}
$$

Therefore, the Laplace transform for the ruin time $\tau_{\lambda}^{m, a}$ is

$$
\begin{aligned}
\mathbb{E}_{x_{0}} \mathrm{e}^{-\delta \tau_{\lambda}^{m, a}} & =\mathbb{P}_{x_{0}}\left\{\tau_{\lambda}^{m, a}<e_{\delta}\right\} \\
& =1-\sum_{i=0}^{m-1} \mathbb{E}_{x_{0}}\left[\frac{\left(\lambda \int_{0}^{e_{\delta}} \mathbf{1}_{\left\{a \leq X_{s}<0\right\}} \mathrm{d} s\right)^{i}}{i !} \exp \left[-\lambda \int_{0}^{e_{\delta}} \mathbf{1}_{\left\{X_{s}<0\right\}} \mathrm{d} s\right]\right] .
\end{aligned}
$$

In order to find the above Laplace transform for the generalized ruin time $\tau_{\lambda}^{m, a}$, we only need to recover the joint Laplace transform $\mathbb{E}_{x_{0}} \exp \left[-\lambda_{1} \int_{0}^{e_{\delta}} \mathbf{1}_{\left\{a \leq X_{s}<0\right\}} \mathrm{d} s-\lambda_{2} \int_{0}^{e_{\delta}^{\lambda}} \mathbf{1}_{\left\{X_{s}<a\right\}} \mathrm{d} s\right]$, which can be found by Theorem 3.3. 
Remark 4.1. Note that this generalized risk model with random observations was first introduced in [2] and [3] for compound Poisson risk processes with independent Erlang $(n)$ interobservation times. Our model with exponential interarrival times is a special case with $n=1$. Unfortunately, it is not apparent that the ruin probability for this general risk model with Erlang $(n)$ interarrival times in [2] and [3] can be associated to the occupation times in a nice way due to the lack-of-memoryless property.

\subsection{The omega risk process}

Let $X$ be the time-homogeneous diffusion process defined in (2.1) with initial value $x_{0}>0$. The so-called omega model was first introduced in [4]. We refer the reader to [1] and [12] for more recent results on the omega model. The event of bankruptcy is introduced for such a model. In contrast to traditional ruin, the bankruptcy is not immediate even if the surplus is negative. To specify the bankruptcy probability, a bankruptcy rate function $\omega(x) \geq 0, x \leq 0$, is introduced, which is typically a decreasing function. Then, whenever the surplus is at level $x \leq 0, \omega(x) \mathrm{d} t$ is the probability of bankruptcy within $\mathrm{d} t$ time units. To be more precise, we can define an auxiliary process $N$ on the same probability space as $X$ so that, given $X$, process $N$ follows the law of a Poisson process with time-dependent intensity $\omega\left(X_{s}\right) \mathbf{1}_{\left\{X_{s}<0\right\}}, s>0$, i.e. $N$ is a Cox process. We then define the bankruptcy time, denoted by $\tau_{\omega}$, as the first arrival time for $N$. One can also simply define

$$
\tau_{\omega}=\inf \left\{t \geq 0: \int_{0}^{t} \omega\left(X_{s}\right) \mathrm{d} s>e_{1}\right\}
$$

for an independent rate-one exponential random variable $e_{1}$; see [17]. Then the risk model considered in [15] corresponds to the omega risk process with a constant rate function $\omega$.

The probability of bankruptcy for the omega risk process can be determined as follows: for any $t>0$,

$$
\mathbb{P}_{x_{0}}\left\{\tau_{\omega}>t\right\}=\mathbb{P}_{x_{0}}\left\{N_{t}=0\right\}=\mathbb{E}_{x_{0}} \exp \left[-\int_{0}^{t} \omega\left(X_{s}\right) \mathbf{1}_{\left\{X_{s}<0\right\}} \mathrm{d} s\right] .
$$

Therefore, the Laplace transform for the bankruptcy time $\tau_{\omega}$ is

$$
\mathbb{E}_{x_{0}} \mathrm{e}^{-\delta \tau_{\omega}}=\mathbb{P}_{x_{0}}\left\{\tau_{\omega}<e_{\delta}\right\}=1-\mathbb{E}_{x_{0}} \exp \left[-\int_{0}^{e_{\delta}} \omega\left(X_{s}\right) \mathbf{1}_{\left\{X_{s}<0\right\}} \mathrm{d} s\right] .
$$

For a constant bankruptcy rate $\omega(x) \equiv \omega$, the bankruptcy probability of the omega risk process coincides with the ruin probability of the risk process with random observations, which has been pointed out in [12]. Therefore, by (4.1) and (4.2),

$$
\mathbb{E}_{x_{0}} \mathrm{e}^{-\delta \tau_{\omega}}=1-\mathbb{E}_{x_{0}} \exp \left[-\omega \int_{0}^{e_{\delta}} \mathbf{1}_{\left\{X_{s}<0\right\}} \mathrm{d} s\right]=\frac{\omega}{\delta+\omega} \frac{g_{-, \delta}\left(x_{0}\right)}{g_{-, \delta}(0)} \frac{\psi_{\delta+\omega}^{+}(0)}{\psi_{\delta+\omega}^{+}(0)+\psi_{\delta}^{-}(0)}
$$

For a piecewise-constant bankruptcy rate

$$
\omega(x)=\lambda_{1} \mathbf{1}_{\{a \leq x<0\}}+\lambda_{2} \mathbf{1}_{\{-\infty<x<a\}} \quad \text { for some } a<0 ;
$$

the corresponding Laplace transform (4.2) can be found by Theorem 3.3.

The omega risk process with rate function $\omega(x)=\lambda \mathbf{1}_{\{a \leq x<0\}}+\infty \mathbf{1}_{\{-\infty<x<a\}}$ is of particular interest. For this process, bankruptcy occurs at rate $\lambda$ when the surplus is between $a$ and 0 , and bankruptcy occurs immediately once the surplus reaches level $a$. It can also be treated as a Chapters 7 and 11 type ruin of [16] for the current model. For such an omega risk process 
with diffusion surplus process, the Laplace transform for the bankruptcy time is given by

$$
\begin{aligned}
\lim _{\lambda_{2} \rightarrow \infty} & \mathbb{E}_{x_{0}} \exp \left[-\lambda \int_{0}^{e_{\delta}} \mathbf{1}_{\left\{a \leq X_{s}<0\right\}} \mathrm{d} s-\lambda_{2} \int_{0}^{e_{\delta}} \mathbf{1}_{\left\{X_{s}<a\right\}} \mathrm{d} s\right] \\
= & \mathbb{E}_{x_{0}}\left[\exp \left[-\lambda \int_{0}^{e_{\delta}} \mathbf{1}_{\left\{a \leq X_{s}<0\right\}} \mathrm{d} s\right] ; e_{\delta}<T_{a}\right],
\end{aligned}
$$

which can be found by Corollary 3.8 .

\section{Examples}

We apply the results in Section 3 to two examples in order to obtain more explicit expressions and to compare them with the known results.

\subsection{Brownian motion with drift}

Let $X_{t}=\mu t+W_{t}$ be a Brownian motion with drift. The corresponding differential equation (2.3) is reduced to

$$
\frac{1}{2} g^{\prime \prime}(x)+\mu g^{\prime}(x)=r g(x), \quad r>0 .
$$

Defining

$$
\beta_{r}^{ \pm}=-\mu \pm \sqrt{\mu^{2}+2 r}
$$

we have

$$
g_{ \pm, r}(x)=\mathrm{e}^{\beta_{r}^{ \pm} x} \quad \text { and } \quad \psi_{r}^{ \pm}(\cdot) \equiv \pm \beta_{r}^{ \pm} .
$$

Moreover,

$$
\begin{aligned}
f_{r}(y, z) & =\mathrm{e}^{\beta_{r}^{-} y+\beta_{r}^{+} z}-\mathrm{e}^{\beta_{r}^{-} z+\beta_{r}^{+} y}, \\
f_{1, r}(y, z) & =\beta_{r}^{-} \mathrm{e}^{\beta_{r}^{-} y+\beta_{r}^{+} z}-\beta_{r}^{+} \mathrm{e}^{\beta_{r}^{-} z+\beta_{r}^{+} y}, \\
f_{2, r}(y, z) & =\beta_{r}^{+} \mathrm{e}^{\beta_{r}^{-} y+\beta_{r}^{+} z}-\beta_{r}^{-} \mathrm{e}^{\beta_{r}^{-} z+\beta_{r}^{+} y},
\end{aligned}
$$

and

$$
f_{12, r}(y, z)=-2 r \mathrm{e}^{\beta_{r}^{-} y+\beta_{r}^{+} z}+2 r \mathrm{e}^{\beta_{r}^{-} z+\beta_{r}^{+} y} .
$$

By Theorem 3.2, we obtain

$$
\begin{aligned}
\mathbb{E} \exp \left[-\lambda_{1} \int_{0}^{e_{\delta}} \mathbf{1}_{\left\{X_{s}<0\right\}} \mathrm{d} s-\lambda_{2} \int_{0}^{e_{\delta}} \mathbf{1}_{\left\{X_{s}>b\right\}} \mathrm{d} s\right] \\
=\frac{C_{1} \mathrm{e}^{\beta_{\delta}^{+} b}+C_{2} \mathrm{e}^{\beta_{\delta}^{-} b}+\lambda_{2}\left(\beta_{\delta}^{+}-\beta_{\delta}^{-}\right) \beta_{\delta+\lambda_{2}}^{-} /\left(\delta+\lambda_{2}\right)}{D_{1} \mathrm{e}^{\beta_{\delta}^{+} b}+D_{2} \mathrm{e}^{\beta_{\delta}^{-} b}},
\end{aligned}
$$

where

$$
\begin{aligned}
& C_{1}=\frac{\delta}{\delta+\lambda_{1}} \beta_{\delta}^{+} \beta_{\delta+\lambda_{1}}^{+}+\beta_{\delta}^{-} \beta_{\delta+\lambda_{2}}^{-}-\frac{\delta}{\delta+\lambda_{1}} \beta_{\delta+\lambda_{1}}^{+} \beta_{\delta+\lambda_{2}}^{-}+2 \delta, \\
& C_{2}=-\frac{\delta}{\delta+\lambda_{1}} \beta_{\delta}^{-} \beta_{\delta+\lambda_{1}}^{+}-\beta_{\delta}^{+} \beta_{\delta+\lambda_{2}}^{-}+\frac{\delta}{\delta+\lambda_{1}} \beta_{\delta+\lambda_{1}}^{+} \beta_{\delta+\lambda_{2}}^{-}-2 \delta, \\
& D_{1}=\beta_{\delta}^{+} \beta_{\delta+\lambda_{1}}^{+}+\beta_{\delta}^{-} \beta_{\delta+\lambda_{2}}^{-}-\beta_{\delta+\lambda_{1}}^{+} \beta_{\delta+\lambda_{2}}^{-}+2 \delta,
\end{aligned}
$$

and

$$
D_{2}=-\beta_{\delta}^{-} \beta_{\delta+\lambda_{1}}^{+}-\beta_{\delta}^{+} \beta_{\delta+\lambda_{2}}^{-}+\beta_{\delta+\lambda_{1}}^{+} \beta_{\delta+\lambda_{2}}^{-}-2 \delta .
$$


By Theorem 3.3, we obtain

$$
\begin{aligned}
\mathbb{E} \exp \left[-\lambda_{1} \int_{0}^{e_{\delta}} \mathbf{1}_{\left\{a \leq X_{s}<0\right\}} \mathrm{d} s-\lambda_{2} \int_{0}^{e_{\delta}} \mathbf{1}_{\left\{X_{s}<a\right\}} \mathrm{d} s\right] \\
=\frac{C_{3} \mathrm{e}^{\beta_{\delta+\lambda_{1}}^{+} a}+C_{4} \mathrm{e}^{\beta_{\delta+\lambda_{1}}^{-} a}+\delta\left(\lambda_{1}-\lambda_{2}\right)\left(\beta_{\delta+\lambda_{1}}^{+}-\beta_{\delta+\lambda_{1}}^{-}\right) \beta_{\delta+\lambda_{2}}^{+} /\left(\delta+\lambda_{1}\right)\left(\delta+\lambda_{2}\right)}{D_{3} \mathrm{e}^{\beta_{\delta+\lambda_{1}}^{+} a}+D_{4} \mathrm{e}^{\beta_{\delta+\lambda_{1}}^{-} a}},
\end{aligned}
$$

where

$$
\begin{aligned}
& C_{3}=-\beta_{\delta}^{-} \beta_{\delta+\lambda_{1}}^{+}+\beta_{\delta}^{-} \beta_{\delta+\lambda_{2}}^{+}-\frac{\delta}{\delta+\lambda_{1}} \beta_{\delta+\lambda_{1}}^{-} \beta_{\delta+\lambda_{2}}^{+}-2 \delta, \\
& C_{4}=\beta_{\delta}^{-} \beta_{\delta+\lambda_{1}}^{-}-\beta_{\delta}^{-} \beta_{\delta+\lambda_{2}}^{+}+\frac{\delta}{\delta+\lambda_{1}} \beta_{\delta+\lambda_{1}}^{+} \beta_{\delta+\lambda_{2}}^{+}+2 \delta, \\
& D_{3}=-\beta_{\delta}^{-} \beta_{\delta+\lambda_{1}}^{+}+\beta_{\delta}^{-} \beta_{\delta+\lambda_{2}}^{+}-\beta_{\delta+\lambda_{1}}^{-} \beta_{\delta+\lambda_{2}}^{+}-2\left(\delta+\lambda_{1}\right),
\end{aligned}
$$

and

$$
D_{4}=\beta_{\delta}^{-} \beta_{\delta+\lambda_{1}}^{-}-\beta_{\delta}^{-} \beta_{\delta+\lambda_{2}}^{+}+\beta_{\delta+\lambda_{1}}^{+} \beta_{\delta+\lambda_{2}}^{+}+2\left(\delta+\lambda_{1}\right) .
$$

In the following we present several results on the occupation times of $X$ with relatively short expressions.

By Corollary 3.1, we have

$$
\mathbb{E} \exp \left[-\lambda \int_{0}^{\infty} \mathbf{1}_{\left\{X_{s}<0\right\}} \mathrm{d} s\right]=\frac{2 \mu}{\mu+\sqrt{\mu^{2}+2 \lambda}} \quad \text { if } \mu \geq 0,
$$

which agrees with Formula 1.4.3 of [6, p. 255].

By Corollary 3.2, we have

$$
\mathbb{E}_{x} \exp \left[-\lambda \int_{0}^{e_{\delta}} \mathbf{1}_{\left\{X_{s}<0\right\}} \mathrm{d} s\right]= \begin{cases}1-\frac{\lambda}{\delta+\lambda} \frac{\beta_{\delta+\lambda}^{+}}{\beta_{\delta+\lambda}^{+}-\beta_{\delta}^{-}} \mathrm{e}^{\beta_{\delta}^{-} x} & \text { if } x \geq 0, \\ \frac{\delta}{\delta+\lambda}+\frac{\lambda}{\delta+\lambda} \frac{-\beta_{\delta}^{-}}{\beta_{\delta+\lambda}^{+}-\beta_{\delta}^{-}} \mathrm{e}^{\beta_{\delta+\lambda}^{+} x} & \text { if } x \leq 0,\end{cases}
$$

which agrees with Formula 1.4.1 of [6, pp. 254, 255].

By Corollary 3.3,

$$
\mathbb{E} \exp \left[-\lambda_{1} \int_{0}^{e_{\delta}} \mathbf{1}_{\left\{X_{s}<0\right\}} \mathrm{d} s-\lambda_{2} \int_{0}^{e_{\delta}} \mathbf{1}_{\left\{X_{s}>0\right\}} \mathrm{d} s\right]=\frac{2 \delta}{-\beta_{\delta+\lambda_{1}}^{-} \beta_{\delta+\lambda_{2}}^{+}},
$$

which agrees with Formula 1.6.1 of [6, p. 258].

By Corollary 3.4,

$$
\begin{aligned}
& \mathbb{E}\left[\exp \left[-\lambda \int_{0}^{e_{\delta}} \mathbf{1}_{\left\{X_{s}<0\right\}} \mathrm{d} s\right] ; e_{\delta}<T_{b}\right] \\
& \quad=\frac{\left(\delta \beta_{\delta+\lambda}^{+} /(\delta+\lambda)-\beta_{\delta}^{-}\right) \mathrm{e}^{\beta_{\delta}^{+} b}+\left(\beta_{\delta}^{+}-\delta \beta_{\delta+\lambda}^{+} /(\delta+\lambda)\right) \mathrm{e}^{\beta_{\delta}^{-} b}-\beta_{\delta}^{+}+\beta_{\delta}^{-}}{\left(\beta_{\delta+\lambda}^{+}-\beta_{\delta}^{-}\right) \mathrm{e}^{\beta_{\delta}^{+} b}+\left(\beta_{\delta}^{+}-\beta_{\delta+\lambda}^{+}\right) \mathrm{e}^{\beta_{\delta}^{-} b}} .
\end{aligned}
$$


By Corollary 3.7,

$$
\mathbb{E} \exp \left[-\lambda \int_{0}^{\infty} \mathbf{1}_{\left\{a \leq X_{s}<0\right\}} \mathrm{d} s\right]=\frac{\mu \beta_{\lambda}^{+} \mathrm{e}^{\beta_{\lambda}^{+} a}-\mu \beta_{\lambda}^{-} \mathrm{e}^{\beta_{\lambda}^{-} a}}{\left(\mu \beta_{\lambda}^{+}-\lambda\right) \mathrm{e}^{\beta_{\lambda}^{+} a}-\left(\mu \beta_{\lambda}^{-}-\lambda\right) \mathrm{e}^{\beta_{\lambda}^{-} a}} \quad \text { if } \mu \geq 0,
$$

which agrees with Formula 1.7.3 of [6, p. 262].

By Corollary 3.8,

$$
\begin{aligned}
& \mathbb{E}\left[\exp \left[-\lambda \int_{0}^{e_{\delta}} \mathbf{1}_{\left\{a \leq X_{s}<0\right\}} \mathrm{d} s\right] ; e_{\delta}<T_{a}\right] \\
& =\frac{\left(\beta_{\delta}^{-}-\delta \beta_{\delta+\lambda}^{-} /(\delta+\lambda)\right) \mathrm{e}^{\beta_{\delta+\lambda}^{+} a}+\left(\delta \beta_{\delta+\lambda}^{+} /(\delta+\lambda)-\beta_{\delta}^{-}\right) \mathrm{e}^{\beta_{\delta+\lambda}^{-} a}-\delta\left(\beta_{\delta+\lambda}^{+}-\beta_{\delta+\lambda}^{-}\right) /(\delta+\lambda)}{\left(\beta_{\delta}^{-}-\beta_{\delta+\lambda}^{-}\right) \mathrm{e}^{\beta_{\delta+\lambda}^{+} a}+\left(\beta_{\delta+\lambda}^{+}-\beta_{\delta}^{-}\right) \mathrm{e}^{\beta_{\delta+\lambda}^{-} a}}
\end{aligned}
$$

which agrees with Formula 2.7.1(1) of [6, p. 301].

\subsection{Brownian motion with two-valued drift}

Let $X$ be a Brownian motion with two-valued drift, specified by the stochastic differential equation

$$
\mathrm{d} X_{t}=\left(\mu_{L} \mathbf{1}_{(-\infty, 0)}\left(X_{t}\right)-\mu_{R} \mathbf{1}_{(0, \infty)}\left(X_{t}\right)\right) \mathrm{d} t+\mathrm{d} W_{t},
$$

where $\mu_{L}, \mu_{R} \in \mathbb{R}$ and $W_{t}$ is a standard one-dimensional Brownian motion. The Brownian motion with two-valued drift, referred to as refracted Brownian motion, is also of interest to risk theory; see, for example, [5] and [11]. Although the Lipschitz assumption (2.2) for the drift function $\mu(\cdot)=\mu_{L} \mathbf{1}_{(-\infty, 0)}(\cdot)-\mu_{R} \mathbf{1}_{(0, \infty)}(\cdot)$ fails, (5.1) still has a unique strong solution; see [20] and [22]. We refer the reader to [21] for recent work on Brownian motion with two-valued drift.

In this two-valued drift model, for $r>0$, suppose that $g_{-, r}(\cdot)$ and $g_{+, r}(\cdot)$ are two independent, positive, and convex solutions of the equation

$$
\frac{1}{2} g^{\prime \prime}(x)+\left(\mu_{L} \mathbf{1}_{(-\infty, 0)}(x)-\mu_{R} \mathbf{1}_{(0, \infty)}(x)\right) g^{\prime}(x)-\operatorname{rg}(x)=0,
$$

with $g_{-, r}(\cdot)$ strictly decreasing and $g_{+, r}(\cdot)$ strictly increasing. We choose

$$
\begin{aligned}
g_{-, r}(x)= & \exp \left[\left(\mu_{R}-\sqrt{\mu_{R}^{2}+2 r}\right) x\right] \mathbf{1}_{\{x>0\}} \\
+ & {\left[c_{-} \exp \left[\left(-\mu_{L}+\sqrt{\mu_{L}^{2}+2 r}\right) x\right]\right.} \\
& \left.+\left(1-c_{-}\right) \exp \left[\left(-\mu_{L}-\sqrt{\mu_{L}^{2}+2 r}\right) x\right]\right] \mathbf{1}_{\{x<0\}}
\end{aligned}
$$

and

$$
\begin{aligned}
g_{+, r}(x)= & {\left[c_{+} \exp \left[\left(\mu_{R}+\sqrt{\mu_{R}^{2}+2 r}\right) x\right]+\left(1-c_{+}\right) \exp \left[\left(\mu_{R}-\sqrt{\mu_{R}^{2}+2 r}\right) x\right]\right] \mathbf{1}_{\{x>0\}} } \\
& +\exp \left[\left(-\mu_{L}+\sqrt{\mu_{L}^{2}+2 r}\right) x\right] \mathbf{1}_{\{x<0\}},
\end{aligned}
$$


where

$$
\begin{aligned}
c_{-} & =\frac{\mu_{R}-\sqrt{\mu_{R}^{2}+2 r}+\mu_{L}+\sqrt{\mu_{L}^{2}+2 r}}{2 \sqrt{\mu_{L}^{2}+2 r}} \\
\text { and } c_{+} & =\frac{-\mu_{L}+\sqrt{\mu_{L}^{2}+2 r}-\mu_{R}+\sqrt{\mu_{R}^{2}+2 r}}{2 \sqrt{\mu_{R}^{2}+2 r}},
\end{aligned}
$$

so that the functions $g_{ \pm, r}(\cdot)$ are differentiable at 0 . Hence, it is easy to see that

$$
\psi_{r}^{-}(0)=-\frac{g_{-, r}^{\prime}(0)}{g_{-, r}(0)}=-\mu_{R}+\sqrt{\mu_{R}^{2}+2 r} \quad \text { and } \quad \psi_{r}^{+}(0)=\frac{g_{+, r}^{\prime}(0)}{g_{+, r}(0)}=-\mu_{L}+\sqrt{\mu_{L}^{2}+2 r} \text {. }
$$

By Theorem 3.1, we obtain

$$
\begin{aligned}
\mathbb{E} \exp \left[-\lambda \int_{0}^{e_{\delta}} \mathbf{1}_{\left\{X_{s}<0\right\}} \mathrm{d} s\right] & =\frac{\delta \psi_{\delta+\lambda}^{+}(0) /(\delta+\lambda)+\psi_{\delta}^{-}(0)}{\psi_{\delta+\lambda}^{+}(0)+\psi_{\delta}^{-}(0)} \\
& =\frac{\delta\left(-\mu_{L}+\sqrt{\mu_{L}^{2}+2(\delta+\lambda)}\right) /(\delta+\lambda)-\mu_{R}+\sqrt{\mu_{R}^{2}+2 \delta}}{-\mu_{L}+\sqrt{\mu_{L}^{2}+2(\delta+\lambda)}-\mu_{R}+\sqrt{\mu_{R}^{2}+2 \delta}}
\end{aligned}
$$

and

$$
\begin{aligned}
\mathbb{E} \exp \left[-\lambda \int_{0}^{e_{\delta}} \mathbf{1}_{\left\{X_{s}>0\right\}} \mathrm{d} s\right] & =\frac{\delta \psi_{\delta+\lambda}^{-}(0) /(\delta+\lambda)+\psi_{\delta}^{+}(0)}{\psi_{\delta+\lambda}^{-}(0)+\psi_{\delta}^{+}(0)} \\
& =\frac{\delta\left(-\mu_{R}+\sqrt{\mu_{R}^{2}+2(\delta+\lambda)}\right) /(\delta+\lambda)-\mu_{L}+\sqrt{\mu_{L}^{2}+2 \delta}}{-\mu_{R}+\sqrt{\mu_{R}^{2}+2(\delta+\lambda)}-\mu_{L}+\sqrt{\mu_{L}^{2}+2 \delta}} .
\end{aligned}
$$

We have thus recovered Equation (15) of [21]. Furthermore, we can obtain the probability density functions for the occupation times $\int_{0}^{t} \mathbf{1}_{\left\{X_{s}<0\right\}}$ and $\int_{0}^{t} \mathbf{1}_{\left\{X_{s}>0\right\}}$ by inverting the corresponding Laplace transform as in [21].

In addition, by (5.2) and (5.3), we can easily derive all the joint Laplace transforms in Theorems 3.2 and 3.3 for Brownian motion with two-valued drift.

\section{Acknowledgement}

The authors thank an anonymous referee for stimulating remarks.

\section{References}

[1] Albrecher, H. and Lautscham, V. (2013). From ruin to bankruptcy for compound Poisson surplus processes. ASTIN Bull. 43, 213-243.

[2] Albrecher, H., Cheung, E. C. K. and Thonhauser, S. (2011). Randomized observation periods for the compound Poisson risk model dividends. ASTIN Bull. 41, 645-672.

[3] Albrecher, H., Cheung, E. C. K. and Thonhauser, S. (2013). Randomized observation periods for the compound Poisson risk model: the discounted penalty function. Scand. Actuarial J. 2013, 424-452.

[4] Albrecher, H., Gerber, H. U. and Shiu, E. S. W (2011). The optimal dividend barrier in the gamma-omega model. Europ. Actuarial J. 1, 43-55. 
[5] Asmussen, S. and Taksar, M. (1997). Controlled diffusion models for optimal dividend pay-out. Insurance Math. Econom. 20, 1-15.

[6] Borodin, A. N. and SAlminen, P. (2002). Handbook of Brownian Motion-Facts and Formulae, 2nd edn. Birkhäuser, Basel.

[7] CaI, N., Chen, N. And Wan, X. (2010). Occupation times of jump-diffusion processes with double exponential jumps and the pricing of options. Math. Operat. Res. 35, 412-437.

[8] Darling, D. A. And Siegert, A. J. F. (1953). The first passage problem for a continuous Markov process. Ann. Math. Statist. 24, 624-639.

[9] Dos ReIs, A. E (1993). How long is the surplus below zero? Insurance Math. Econom. 12, 23-38.

[10] Feller, W. (1954). Diffusion processes in one dimension. Trans. Amer. Math. Soc. 77, 1-31.

[11] Gerber, H. U. And ShiU, E. S. W. (2006). On optimal dividends: from reflection to refraction. J. Comput. Appl. Math. 186, 4-22.

[12] Gerber, H. U., Shiu, E. S. W. And Yang, H. (2012). The Omega model: from bankruptcy to occupation times in the red. Europ. Actuarial J. 2, 259-272.

[13] Gīhman, Ĭ. İ. and Skorohod, A. V. (1972). Stochastic Differential Equations. Springer, New York.

[14] Kyprianou, A. E., Pardo, J. C. And Pérez, J. L. (2012). Occupation times of refracted Lévy processes. Preprint. Available at http://arxiv.org/abs/1205.0756v1.

[15] Landriault, D., Renaud, J.-F. and Zhou, X. (2011). Occupation times of spectrally negative Lévy processes with applications. Stoch. Process. Appl. 121, 2629-2641.

[16] LI, B., TANG, Q. AND ZHOU, X. (2013). Liquidation risk in the presence of chapter 7 and chapter 11 of the U.S. bankruptcy code. Submitted.

[17] Loeffen, R. L., Renaud, J.-F. And Zhou, X.(2013). Occupation times of intervals until first passage times for spectrally negative Lévy processes. To appear in Stoch. Process. Appl.

[18] Pitman, J. AND YoR, M. (1999). Laplace transforms related to excursions of a one-dimensional diffusion. Bernoulli 5, 249-255.

[19] Pitman, J. And Yor, M. (2003). Hitting, occupation and inverse local times of one-dimensional diffusions: martingale and excursion approaches. Bernoulli 9, 1-24.

[20] Prokhorov, Y. V. And Shiryaev, A. N. (eds) (1998). Probability theory III. Stochastic calculus. Springer, Berlin.

[21] Simpson, D. J. W. And Kuske, R. (2013). The positive occupation time of Brownian motion with two-valued drift and asymptotic dynamics of sliding motion with noise. Preprint. Available at http://arxiv.org/abs/1204.5985v2.

[22] Stroock, D. W. and Varadhan, S. R. S. (1969). Diffusion processes with continuous coefficients. I. Commun. Pure Appl. Math. 22, 345-400.

[23] Yin, C. And Yuen, K. (2013). Some exact joint laws associated with spectrally negative Lévy processes and applications to insurance risk theory. Preprint. Available at http://arxiv.org/abs1101.0445v3.

[24] Zhang, C. AND Wu, R. (2002). Total duration of negative surplus for the compound Poisson process that is perturbed by diffusion. J. Appl. Prob. 39, 517-532. 